\title{
On the behaviour of organised disturbances in a turbulent boundary-layer
}

\author{
P K SEN and SRINIVAS V VEERAVALLI \\ Department of Applied Mechanics, Indian Institute of Technology, \\ New Delhi 110016, India \\ MS received 29 March 1997; revised 17 July 1997
}

\begin{abstract}
This paper is in continuation of our earlier work on the role of hydrodynamic stability theory in understanding wall-bounded turbulent flows. Work in this area was pioneered by Malkus, followed by Reynolds, Tiederman and Hussain. Numerical results showed that the linear instability modes are damped, a result also confirmed by our earlier work for the boundary layer flow case. This led to waning of interest in this approach.

In the present work the problem is reformulated using an improved nonisotropic model for the stress tensor based on the model of Pope. This improved model does yield unstable wall modes. A wide range of unstable wavenumbers is observed and these unstable modes mimic some of the key features of wall-bounded turbulent flows. Comparisons with experimental data are also presented. The present work keeps alive the question of relevance of stability theory to wall-bounded turbulent flows.
\end{abstract}

Keywords. Hydrodynamic stability; wall-bounded turbulent flow; boundary layer flow; non-isotropic model.

\section{Introduction}

The motivation for the present paper is primarily to re-examine a question that has been asked before, but has got mixed answers so far. Whether or not hydrodynamic stability theory has any bearing on the problem of wall-bounded turbulent shear flows, especially in view of the fact that it does have a significant role in free turbulent shear flows. See, for example, the discussion by Gaster et al (1985) on the turbulent mixing layer, the comprehensive review on stability and free turbulent shear flows by Liu (1988), and a more recent overview by Roshko (1992).

The question of a connection between stability theory and turbulent shear flow was first raised by Landau (1944) based on a nonlinear stability model. While his work did not prove to be a suitable model for turbulence, his equation for nonlinear growth found 
many applications in the field of instability and transition. The next work of great conceptual importance is that of Malkus (1956), wherein a theory of turbulence was developed based on the introduced concept of marginal stability. According to this concept, it was proposed that if the mean velocity profile typical of wall-bounded turbulent flows is used in the solution of the classical Orr-Sommerfeld equation, then the profile would prove to be marginally or neutrally stable at the existing flow Reynolds number. It is significant also that Malkus suggested that the molecular viscosity and not the eddy viscosity be used in the solution of the Orr-Sommerfeld equation. Malkus's theory was rigorously put to test in an important work by Reynolds \& Tiederman (1967). This work also gives a lucid review of Malkus's theory. Reynolds \& Tiederman (1967) investigated the stability of fully developed turbulent flow between parallel plates, on the lines of Malkus's proposed theory. They used the turbulent mean velocity profile for channel flow in the solution of the classical Orr-Sommerfeld equation (using the molecular viscosity in the Orr-Sommerfeld equation). The results obtained showed without doubt that Malkus's theory as proposed was not valid, and there was a huge discrepancy between the flow Reynolds number and the Reynolds number corresponding to neutral or marginal stability, the latter being an order of magnitude higher than the former. Prabhu (1968, private communication) also independently obtained the same result. More recently, Sen et al (1993) obtained the same result for the turbulent boundary layer flow problem.

Although Malkus's theory was not proven, it did not immediately detract attention from the general question of connection between instability and turbulence, and, as mentioned before, considerable success was obtained later on in linking the two for the cases of free turbulent shear flows. The results for free turbulent shear flows were very decisive mainly because of the inflectional mean-velocity profile present in such flows. Thus the dominant instability is the inviscid instability, which continues to exist even in the presence of the existing turbulent flow field. Also, the dominant eddy structure of the turbulent flow field is found to correlate well with the vorticular structure corresponding to the inviscid instability (see Liu 1988 and Roshko 1992 for comprehensive reviews).

The general question of connection between instability and turbulence, with reference to wall-bounded turbulent shear flow, went through another round of serious examination by Reynolds \& Hussain (1972). This time, abandoning Malkusian precepts, first the basic equations were derived for a superposed organised disturbance in turbulent flow. Thereafter an equation for the stability of this organised disturbance was derived, which was like an augmented form of the classical Orr-Sommerfeld equation, but containing extension terms dependent on the eddy viscosity. Underlying the model was a closure problem for the Reynolds stress tensor, which was resolved based on what the authors called the "Newtonian eddy' model. Results of solution of their extended Orr-Sommerfeld equation, for channel flow, again yielded damped modes. Nevertheless, the results were closer to establishing a connection between instability and turbulent wall flows than was obtained in Reynolds \& Tiederman (1967). Experiments performed by Hussain \& Reynolds $(1970,1972)$ showed some agreement with the theory of Reynolds \& Hussain (1972). Subsequent to the works of Reynolds \& Hussain (1972), some nonlinear and three-dimensional theories were also developed (see for example the work of Jang et al 1986), but these are outside the scope of the present discussion. 
The present work seeks to re-examine the question of connection between instability and wall turbulence. The organised disturbances are linear and two-dimensional, and the particular flow chosen is the turbulent boundary layer flow over a flat plate. The chief improvement over the work of Reynolds \& Hussain (1972) is that a more realistic and improved model is chosen for the turbulent stress tensor, based on the anisotropic model of Pope (1975). This model gives further extensions of the Orr-Sommerfeld equation over what Reynolds \& Hussain (1972) obtained. The results show that an unstable wallmode exists over a wide range of the spatial wavenumber $\alpha$. The instability characteristics scale very well with the inner variables of turbulent flow. Also the organised disturbances mimic some of the key features of wall-turbulence. Experiments performed for the one dimensional energy-spectrum for the turbulent longitudinal velocity, indicate that the range of unstable wavelengths is well contained within the energy-containing part of the energy spectrum. Thus, the present work keeps alive the question of relevance of stability theory in understanding turbulence in wall-bounded turbulent flows.

Preliminary versions of the present work appeared in Sen \& Veeravalli $(1994,1996)$.

\section{Theory}

The instantaneous velocity vector $u_{i}$ obeys the Navier-Stokes and continuity equations:

$$
\begin{aligned}
& \frac{\partial u_{i}}{\partial t}+u_{j} \frac{\partial u_{i}}{\partial x_{j}}=-\frac{1}{\rho} \frac{\partial p}{\partial x_{i}}+v \frac{\partial^{2} u_{i}}{\partial x_{j} \partial x_{j}} \\
& \frac{\partial u_{i}}{\partial x_{i}}=0
\end{aligned}
$$

The velocity and pressure fields are decomposed in turbulent flows by the well known Reynolds decomposition; typically,

$$
u_{i}=\bar{u}_{i}+u_{i}^{\prime} ; \quad p=\bar{p}+p^{\prime} .
$$

Here $\bar{u}_{i}, \bar{p}$ are respectively the mean velocity and pressure, and $u_{i}^{\prime}, p^{\prime}$ are the (random) fluctuations. If we now superpose an organised (solenoidal) disturbance $\tilde{u}_{i}, \tilde{p}$ (with zero mean), the instantaneous velocity and pressure are respectively given as follows:

$$
u_{i}=\bar{u}_{i}+\tilde{u}_{i}+u_{i}^{\prime} ; \quad p=\bar{p}+\tilde{p}+p^{\prime} .
$$

The time averages of $u_{i}, p$ are still respectively $\bar{u}_{i}, \bar{p}$, but the ensemble (phase-locked) averages are different. The ensemble averages are given respectively as

$$
\left\langle u_{i}\right\rangle=\bar{u}_{i}+\tilde{u}_{i} ; \quad\langle p\rangle=\bar{p}+\tilde{p} .
$$

Moreover, the organised disturbance is assumed to be small, or linear, and in addition it obeys the following:

$$
\left|\left\langle\tilde{u}_{i} \tilde{u}_{j}\right\rangle\right| \ll\left|\left\langle u_{i}^{\prime} u_{j}^{\prime}\right\rangle\right|
$$

The above assumption (4) restricts the organised disturbances to being weaker than was considered by Reynolds \& Hussain (1972). 
For future clarity some definitions and notations are introduced: (i) an overbar (-) over any quantity implies time average; (ii) the symbols \langle\rangle enclosing a quantity denote ensemble average. Further, we note that: (i) when the flow field is not perturbed by an organised disturbance, the time average and the ensemble average are the same; (ii) when the flow field contains an organised disturbance, the time average and the ensemble average are different; (iii) it will be seen later that if the organised disturbance in the flow field is small (in the sense defined in (4) above), then the time average is the same as the time average of the corresponding flow field that is not perturbed by the organised disturbance. Hereafter, the terms 'perturbed flow' and 'unperturbed flow' will mean respectively the flow that is perturbed and that is not perturbed, by the organised disturbance.

One needs now to look into the forms that (1a) takes upon different ways of averaging. First the typical time average, with the disturbance decomposition as in (2), which yields the well-known Reynolds averaged equation:

$$
\frac{\partial \bar{u}_{i}}{\partial t}+\bar{u}_{j} \frac{\partial \bar{u}_{i}}{\partial x_{j}}=-\frac{1}{\rho} \frac{\partial \bar{p}}{\partial x_{i}}+v \frac{\partial^{2} \bar{u}_{i}}{\partial x_{j} \partial x_{j}}-\frac{\partial\left(\overline{u_{i}^{\prime} u_{j}^{\prime}}\right)}{\partial x_{j}} .
$$

One can also obtain the ensemble average of (1a), to obtain the equation for $\left\langle u_{i}\right\rangle$. We need to remember the following rules for ensemble averaging, given with respect to any two generic quantities $a, b$.

$$
\langle\bar{a} \tilde{b}\rangle=\bar{a} \tilde{b} ; \quad\left\langle a^{\prime} \tilde{b}\right\rangle=0
$$

and, in view of (4), and in the event that $\tilde{a}, \tilde{b} \sim O\left(\tilde{u}_{i}\right)$, then the following is also valid:

$$
\langle\tilde{a} \tilde{b}\rangle \approx 0 ; \quad \overline{\tilde{a} \tilde{b}} \approx 0 .
$$

In view of (4) and (7), it may be noted that the time average of (1a), even when disturbance form (3a) is being considered, becomes the same as (5). Particularly, the organised disturbances are assumed small enough not to alter $\overline{u_{i}^{\prime} u_{j}^{\prime}}$. Further discussion on this point will be taken up later.

Next we look at the form of (1a) after ensemble averaging, with the disturbance decomposition as in (3a). Remembering (6) and (7) one obtains:

$$
\frac{\partial\left\langle u_{i}\right\rangle}{\partial t}+\left\langle u_{j}\right\rangle \frac{\partial\left\langle u_{i}\right\rangle}{\partial x_{j}}=-\frac{1}{\rho} \frac{\partial\langle p\rangle}{\partial x_{i}}+v \frac{\partial^{2}\left\langle u_{i}\right\rangle}{\partial x_{j} \partial x_{j}}-\frac{\partial\left(\left\langle u_{i}^{\prime} u_{j}^{\prime}\right\rangle\right)}{\partial x_{j}} .
$$

One is now in a position to obtain the dynamic equation for the organised disturbance, by subtracting (5) from (8), and remembering (3b). This gives the following equation:

$$
\frac{\partial \tilde{u}_{i}}{\partial t}+\bar{u}_{j} \frac{\partial \tilde{u}_{i}}{\partial x_{j}}+\tilde{u}_{j} \frac{\partial \bar{u}_{i}}{\partial x_{j}}=-\frac{1}{\rho} \frac{\partial \tilde{p}}{\partial x_{i}}+v \frac{\partial^{2} \tilde{u}_{i}}{\partial x_{j} \partial x_{j}}+\frac{\partial \tilde{r}_{i j}}{\partial x_{j}},
$$

where $\tilde{r}_{i j}$ is the difference between the ensemble average and time average of $u_{i}^{\prime} u_{j}^{\prime}$, that is it is the modulation in the Reynolds stress tensor, and is given as follows:

$$
\tilde{r}_{i j} \equiv-\left(\left\langle u_{i}^{\prime} u_{j}^{\prime}\right\rangle-\overline{u_{i}^{\prime} u_{j}^{\prime}}\right) \text {. }
$$

Reynolds \& Hussain (1972) have shown that $\tilde{r}_{i j}$ is of the order $\tilde{r}_{i j} \sim O\left(\tilde{u}_{i}\right)$. The above equations pose a closure problem for the various forms of the turbulent stresses, including 


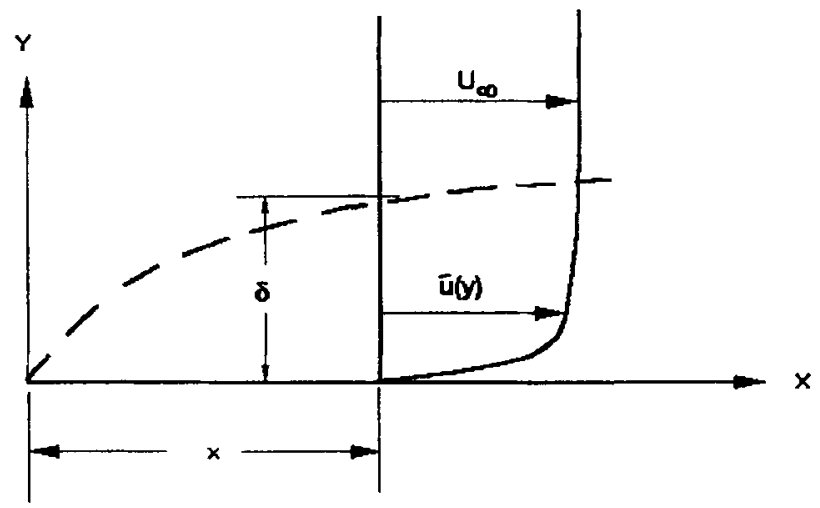

Figure 1. Definition sketch for the flat plate turbulent boundary-layer.

$\tilde{r}_{i j}$. It will be instructive to look at simplified physical models and reasoning to understand the manner of resolution proposed for the closure problems.

At this stage we introduce (twice) the rate of strain tensor and (twice) the vorticity tensor, respectively $s_{i j}$ and $\omega_{i j}$, (in generic form) as follows:

$$
s_{i j}=\left(\frac{\partial u_{i}}{\partial x_{j}}+\frac{\partial u_{j}}{\partial x_{i}}\right) ; \quad \omega_{i j}=\left(\frac{\partial u_{i}}{\partial x_{j}}-\frac{\partial u_{j}}{\partial x_{i}}\right)
$$

The expressions in (11) are in generic form, implying that if, for example, $u$ is replaced by $\bar{u}$, then $s_{i j}$ and $\omega_{i j}$ are respectively replaced by $\bar{s}_{i j}$ and $\bar{\omega}_{i j}$.

Let us now restrict our attention to 2-D near-parallel flows, specifically the turbulent boundary layer. A definition sketch is shown in figure 1 . Note that in the discussions to follow, the vectors $\left(u_{1}, u_{2}, u_{3}\right)$ and $(u, v, w)$, will be used interchangably and so also $\left(x_{1}, x_{2}, x_{3}\right)$ and $(x, y, z)$. The $x$ coordinate is in the direction of the free stream, $y$ is in the direction normal to the wall, and $z$ is the transverse direction. Also we make the quasi-parallel assumption due to which the mean-velocity field is given as $\bar{u}=\bar{u}(y)$, $\bar{v} \approx 0$, and $\bar{w}=0$. Further, all the mean-velocity gradients, except for $\partial \bar{u} / \partial y$, are either zero, or negligibly small. The turbulence $\left(u_{i}^{\prime}\right)$-field is also assumed homogeneous in the $z$ direction, and near-homogeneous in the $x$ direction. All derivatives of time-averaged quantities are zero in the $z$-direction, and nearly zero in the $x$-direction. Moreover the correlations $\overline{u^{\prime} w^{\prime}}$ and $\overline{v^{\prime} w^{\prime}}$ are zero. However, $\overline{w^{\prime 2}}$ is non-zero, and this term keeps the Reynolds stress tensor $\overline{u_{i}^{\prime} u_{j}^{\prime}}$ three dimensional. The velocity scale for the problem could be either the free stream velocity, $U_{\infty}$ (outer scaling), or the friction velocity, $v_{*}$ (inner scaling). The relevant length scales are the boundary layer thickness $\delta$ or the displacement thickness $\delta^{*}$ (both outer scaling), or the viscous length scale, $v / v_{*}$ (inner scaling). The characteristic Reynolds number for the problem is $R \equiv U_{\infty} \delta / \nu$.

With the problem posed as above, two closure schemes are presented. The first, called the 'isotropic eddy viscosity model' is identical in form to the Newtonian eddy model of Reynolds \& Hussain (1972). The second, called the 'anisotropic eddy viscosity model' is based on the model proposed by Pope (1975). 


\subsection{The isotropic eddy viscosity model}

The isotropic eddy viscosity model, for the unperturbed flow, is given by the following well-known expression:

$$
-\overline{u_{i}^{\prime} u_{j}^{\prime}}=-\frac{2}{3} k \delta_{i j}+\epsilon \bar{s}_{i j} ; \quad \text { with, } k \equiv \frac{1}{2}\left(\overline{u_{i}^{\prime} u_{i}^{\prime}}\right)
$$

In (12), $k$ is the turbulent kinetic energy, and $\epsilon$ is the eddy viscosity. Also, in the wallbounded two-dimensional flow being considered here, $\epsilon=\epsilon(y)$, that is, the eddy viscosity is a function of the coordinate perpendicular to the wall. Details of the form of $\epsilon$ will be discussed later.

Equation (12), despite similarity in form with the corresponding form for laminar shear flow, is no more believed to represent a gradient-type diffusion of momentum. In fact, the justification for the above form is the following (see Tennekes \& Lumley 1972). First, the eddy viscosity is of the form $\epsilon \sim v^{\prime} l$, where $l$ is a turbulent length scale (which may loosely be called the mixing length for historical reasons). This length scale is unique for wallbounded turbulent shear flows, like in the case of the flat plate, and is given as $l \sim(\kappa y)$ in the inner region and approaching a constant in the outer region. Also $\kappa$ is the Von Karman constant. Second, the turbulent velocity scale is given uniquely by $v_{*}=\left(\tau_{w} / \rho\right)^{1 / 2}$, where $\tau_{w}$ is the wall shear stress; hence $v^{\prime}$ scales with $v_{*}$. Third, the turbulent time scale and the mean flow time scale are of the same order. Thus, form (12) emerges as a dimensional necessity. Since it is not the scope of the present paper to go into a detailed discussion on these points, it suffices to say that form (12) is a valid and legitimate form for wall-bounded shear flows, like the flat plate, that are governed by one velocity scale $v_{*}$ and one length scale $l$.

The ideas developed over the past years, on lines of the above discussion, can be summarised by the proposition of the 'generalised eddy viscosity hypothesis' (GEVH). Such cases of turbulent shear flow that have one characteristic velocity scale and one characteristic length scale of turbulence and, where the turbulence is sustained by the mean shear, will have a time scale of turbulence that is of the same order as the mean flow time scale, the latter being given by the reciprocal of the dominant mean-velocity gradient. Further, the turbulent shear stress tensor in such flows is capable of being described by products of scalar functions derived from the turbulence and appropriate tensorial combinations of the mean-velocity gradients. Further, any description of the shear stress tensor must be tensorially consistent, that is, it must obey the laws of tensor transformation. In actuality, form (12) is the simplest and most widely used manifestation of GEVH.

What is important for us to note, in the context of the present work, is that if (12) be looked upon as a constitutive equation for $\overline{u_{i}^{\prime} u_{j}^{\prime}}$, then the right hand side of this equation has one part that is solely dependent on the existing turbulence, viz. $\epsilon \sim v^{\prime} l$, and the other part on gradients of the mean flow. It is reasonable to assume that if such a flow is perturbed, yet leaving $v^{\prime}$ and $l$ unchanged, then the eddy viscosity $\epsilon$ remains unchanged. In other words, if the existing turbulence is unchanged then $\epsilon$ remains unchanged. Further, if the perturbations cause modulations in the mean field, then these modulations appear as modulations of the Reynolds stress tensor. The organised disturbance does exactly this. First it modulates the mean flow, as may be seen from (3b). Second, it can be seen from Reynolds \& Hussain (1972) that the modification of the turbulent kinetic energy, due to the 
presence of the organised disturbance, is $O\left(\tilde{u}^{2}\right)$. In view of (4) this is negligible, and thus there is no change in the turbulence, and consequently no change in the eddy viscosity.

In the extreme case of a very high-amplitude organised disturbance, possibly even the form (12) would not be valid, since the imposed disturbance would introduce its own length and velocity scales in the problem, thus negating the validity of GEVH.

The consequence of the above discussion is significant, in that (12a) can be extended to the flow modulated by the organised perturbation in the following manner:

$$
-\left\langle u_{i}^{\prime} u_{j}^{\prime}\right\rangle=-\frac{2}{3} k \delta_{i j}+\epsilon\left\langle s_{i j}\right\rangle
$$

It is easy to see now that $\left\langle u_{i}^{\prime} u_{j}^{\prime}\right\rangle$ will be modulated by the organised perturbation despite $\epsilon$ having remained unchanged. It is assumed in (13), following Reynolds \& Hussain (1972) that the organised disturbances do not modulate the dilatational part of the stress tensor. Thus $(\langle k\rangle-k)$ is neglected, where $\langle k\rangle=(1 / 2)\left\langle u_{i}^{\prime} u_{i}^{\prime}\right\rangle$. Besides, even if $(\langle k\rangle-k)$ were to be retained, it would be like the the pressure term in (9), and this would not eventually affect the derivation of the extended Orr-Sommerfeld equation. Further, by subtracting (12a) from (13), it is easy to see that $\tilde{r}_{i j} \sim O\left(\tilde{u}_{i}\right)$, which is consistent with Reynolds \& Hussain (1972), and the expression for $\tilde{r}_{i j}$ is given as follows:

$$
\tilde{r}_{i j}=\epsilon \tilde{s}_{i j}
$$

Reynolds \& Hussain (1972) have not considered $\tilde{u}_{i}$ as small as in (4) herein, nor have they considered very high amplitude perturbations. However, they have reasoned that, with some restrictions, in their problem also the Newtonian eddy model will give the same expression for $\tilde{r}_{i j}$ as in (14).

\subsection{The anisotropic eddy viscosity model}

The necessity of considering an improved model, over the isotropic eddy viscosity model, is quite strong, and various angles of the matter will be looked into. The main reason for proposing an improved model (without keeping any allied problem, like the hydrodynamic stability problem, in view) has been given by Pope (1975). There could be some flows, wherein the turbulence is isotropic or anisotropic, where GEVH is not valid (one such simple example is given by Tennekes \& Lumley (1972), of turbulent flow over a flat-plate with vertical blowing or suction through the plate). Also, there are some flows wherein the turbulence is anisotropic, yet GEVH is valid. The most obvious examples in the latter category are the simple wall turbulent flows, the flat plate boundary layer problem being one of them. The important contribution of Pope (1975) has been to develop advanced level constitutive equations under GEVH, mainly to account for anisotropy in flows that obey GEVH.

First we examine the shortcomings of (12) as a constitutive equation for the Reynolds stress tensor. It can be seen from (12a) that the normal stresses predicted by this model give $\overline{u^{\prime 2}}, \overline{v^{\prime 2}}, \overline{w^{\prime 2}}$ all equal to $\frac{2}{3} k$. This is quite a departure from the known anisotropic behaviour in the entire shear layer and particularly in the wall region. To overcome this shortcoming, the constitutive equation that has been given by Pope (1975), under the aegis of $\mathrm{GEVH}$, is as follows:

$$
-\overline{u_{i}^{\prime} u_{j}^{\prime}}=-\frac{2}{3} k \delta_{i j}+\epsilon \bar{s}_{i j}-\epsilon C\left(k / \epsilon_{d}\right)\left[\frac{1}{2}\left[\bar{\omega}_{i k} \bar{s}_{k j}-\bar{s}_{i k} \bar{\omega}_{k j}\right]\right]
$$


In (15), $\epsilon_{d}$ is the dissipation rate of the turbulent kinetic energy, and $C$ is a constant to match experimental results. The last term in (15) accentuates the anisotropy generally by augmenting $\overline{u^{\prime 2}}$, reducing $\overline{v^{\prime 2}}$, and leaving $\overline{w^{\prime 2}}$ unchanged. Further, the last term leaves $\overline{u^{\prime} v^{\prime}}$ unchanged. Pope (1975) has one more term in (15), involving a scalar invariant of $\bar{s}_{i j}$, namely the scalar product $\bar{s}_{i j} \bar{s}_{j i}$. This term tends to equalise $\overline{u^{\prime 2}}$ and $\overline{v^{\prime 2}}$, but makes $\overline{w^{\prime 2}}$ different from both. This term is rejected for the disturbance equation on two grounds. First, the accentuation of anisotropy in the $z$ direction has not much relevance in the present problem in which basically two-dimensional disturbances are being considered. The second reason is more serious. The $\bar{s}_{i j} \bar{s}_{j i}$-term has to be incorporated artificially with a two-dimensional Kronecker delta. Since the latter does not behave like the identity matrix in three dimensions, it destroys the tensorial symmetry of the problem, and, eventually renders the disturbances non-transformable by Squire's theorem. Pope (1975) himself has suggested that this term can be ignored if accentuation of anisotropy in the $z$ direction is not important. Thus, in the present problem, the $\bar{s}_{i j} \bar{s}_{j i}$-term is nominally considered in the (unperturbed) mean-motion only, mainly to estimate the value of the two anisotropy parameters $C$ and $C_{1}$, the latter to be introduced later.

Equation (15) has a unique tensorial form involving $\bar{s}_{i j}$ and $\bar{\omega}_{i j}$ under GEVH. Over and above the basic isotropic eddy viscosity model, (12), this is the next advanced-level constitutive equation, from amongst the hierarchy of constitutive equations derived by Pope (1975). So long as GEVH is valid, and a model is to be proposed within GEVH, the uniqueness of the form of (15) leaves little scope for seeking out other tensorial forms at the same advanced-level as in (15). At most, one can look for alternative but equivalent forms for the turbulence-derived scalar functions like $k / \epsilon_{d}$. It is therefore reasonable to conclude, that, since the present problem obeys GEVH, form (15) is clearly both a unique and logical improvement over (12). Also, other such turbulence models that are not within the framework of GEVH, are outside the scope of the present paper. But then, such models would not also admit even a form like (12), and it is doubtful whether such models would capture the real physics in the present problem, as much as GEVH would.

Whilst retaining the basic tensorial form as in (15), for certain conveniences in calculations we introduce an alternative but equivalent scalar function in place of the turbulencederived scalar function $k / \epsilon_{d}$ in (15). Now, $k / \epsilon_{d}$ represents a turbulent time scale. It is convenient to put this term equivalently as $k / \epsilon_{d}=\lambda /(\bar{u})^{\prime}$, where $(\bar{u})^{\prime}=(\partial \bar{u} / \partial y)$. The function $\lambda$ is called the anisotropy function, with the constant $C$ in (15) absorbed in $\lambda$. The anisotropy function $\lambda$ thus physically represents the ratio of the turbulent time scale to the mean flow time scale. The constitutive equation adopted in the present problem is therefore given as follows:

$$
-\overline{u_{i}^{\prime} u_{j}^{\prime}}=-\frac{2}{3} k \delta_{i j}+\epsilon \bar{s}_{i j}-\epsilon\left(\lambda / \bar{u}^{\prime}\right)\left[\frac{1}{2}\left[\bar{\omega}_{i k} \bar{s}_{k j}-\bar{s}_{i k} \bar{\omega}_{k j}\right]\right]
$$

Using exactly the same arguments as in $\S 2.1$ earlier, (16) may be extended to perturbed flow, as follows:

$$
-\left\langle u_{i}^{\prime} u_{j}^{\prime}\right\rangle=-\frac{2}{3} k \delta_{i j}+\epsilon\left\langle s_{i j}\right\rangle-\epsilon\left(\lambda / \bar{u}^{\prime}\right)\left[\frac{1}{2}\left[\left\langle\omega_{i k}\right\rangle\left\langle s_{k j}\right\rangle-\left\langle s_{i k}\right\rangle\left\langle\omega_{k j}\right\rangle\right]\right] .
$$

Also, as before, $\tilde{r}_{i j}$ may be obtained by subtracting (16) from (17), and is given as follows:

$$
\tilde{r}_{i j}=\epsilon \tilde{s}_{i j}-\epsilon\left(\lambda / \bar{u}^{\prime}\right)\left[\frac{1}{2}\left[\bar{\omega}_{i k} \tilde{s}_{k j}+\tilde{\omega}_{i k} \bar{s}_{k j}-\bar{s}_{i k} \tilde{\omega}_{k j}-\tilde{s}_{i k} \bar{\omega}_{k j}\right]\right]
$$




\subsection{The anisotropy function}

A word is now in order regarding the anisotropy function $\lambda$, and its efficacy in the present formulation. One can put $\epsilon_{d} \sim \overline{u^{\prime} v^{\prime}}(\bar{u})^{\prime}$ in (16), the expression for $\epsilon_{d}$ becoming almost exact in the outer region where production nearly equals dissipitation. The expression for $\lambda$ is thereafter given as follows:

$$
\lambda=C\left(k /\left(-\overline{u^{\prime} v^{\prime}}\right)\right)
$$

where $C$ is either a constant, or at most a slowly varying function. It is noted from (19) that $\lambda$ is a universal non-dimensional function, and is like the inverse of the structure function given in Townsend (1976), and also plotted by Hinze (1975; figures 7.21 \& 7.22). In fact, since $k / \epsilon_{d}$ in (15) is meant to extract a turbulent time scale, one might as well replace $\epsilon_{d}$ by $\overline{u^{\prime} v^{\prime}}(\bar{u})^{\prime}$ in the entire range of $y$ in $(15)$.

The contribution of the $\bar{s}_{i j} \bar{s}_{j i}$-term, to the (unperturbed) mean-motion, is nominally added to (16) using a form similar to (19) with $C_{1}$ in place of $C$. Thereafter, upon expanding (16), one obtains the following expressions for the turbulence stresses:

$$
\begin{aligned}
-\frac{\overline{u^{\prime 2}}}{k} & =-\frac{2}{3}+0-\left(C+C_{1}\right)\left(\frac{\epsilon(\bar{u})^{\prime}}{-\overline{u^{\prime} v^{\prime}}}\right) ; \\
-\frac{\overline{v^{\prime 2}}}{k} & =-\frac{2}{3}+0+\left(C-C_{1}\right)\left(\frac{\epsilon(\bar{u})^{\prime}}{-\overline{u^{\prime} v^{\prime}}}\right) ; \\
-\frac{w^{\prime 2}}{k} & =-\frac{2}{3}+0+2 C_{1}\left(\frac{\epsilon(\bar{u})^{\prime}}{-\overline{u^{\prime} v^{\prime}}}\right) ; \\
-\frac{\overline{u^{\prime} v^{\prime}}}{k} & =0+\frac{\epsilon(\bar{u})^{\prime}}{k}+0 .
\end{aligned}
$$

The accentuation of anisotropy now becomes clear, and in view of (20d), (20a,b,c) may be rewritten as follows:

$$
\frac{\overline{u^{\prime 2}}}{k}=\frac{2}{3}+C+C_{1} ; \quad \frac{\overline{v^{\prime 2}}}{k}=\frac{2}{3}-C+C_{1} ; \quad \frac{\overline{w^{\prime 2}}}{k}=\frac{2}{3}-2 C_{1} .
$$

Estimation of the values of $C$ and $C_{1}$ have been carried out from Klebanoff's experimental data (cf. Hinze 1975), and from the DNS data of Spalart (1988). Thereafter, using (19), $\lambda$ has been evaluated. The results are shown in figure $2 \mathrm{a}$, in which $y^{+} \equiv y v_{*} / v$ is $y$-scaled in inner variables. It is seen that $C_{1}$ is quite small except very close to the wall, where it has a value of $\simeq 0.2$. In case that $C_{1}=0$, then it is seen from $(21 \mathrm{a}, \mathrm{b}, \mathrm{c})$ that there is a restriction on admissible values of $C$, viz. $C \leq 0.67$, to avoid negative values of $\overline{v^{\prime 2}}$. However, close to the wall, where $C_{1} \simeq 0.2, C$ can have values $\simeq 0.9$, as may be seen in figure $2 \mathrm{a}$.

It is seen in figure $2 \mathrm{a}$ that $\lambda$ has a value of 25 close to the wall, relaxing to around 2 in the region $y^{+} \sim 60$. In the outer region it relaxes further to around 1 , and drops to 0 close to the edge of the boundary layer where there is isotropy in the sense that $\overline{u^{\prime 2}}, \overline{v^{\prime 2}}$, and $\overline{w^{\prime 2}}$ are nearly equal to each other. The relatively high values of $\lambda$ obtained close to the wall are because of the strong anisotropy in the wall region.

Stability calculations were performed with a wide latitude of variation in the form of $\lambda$, retaining the feature that $\lambda$ has a high value in the wall region. It was observed that 

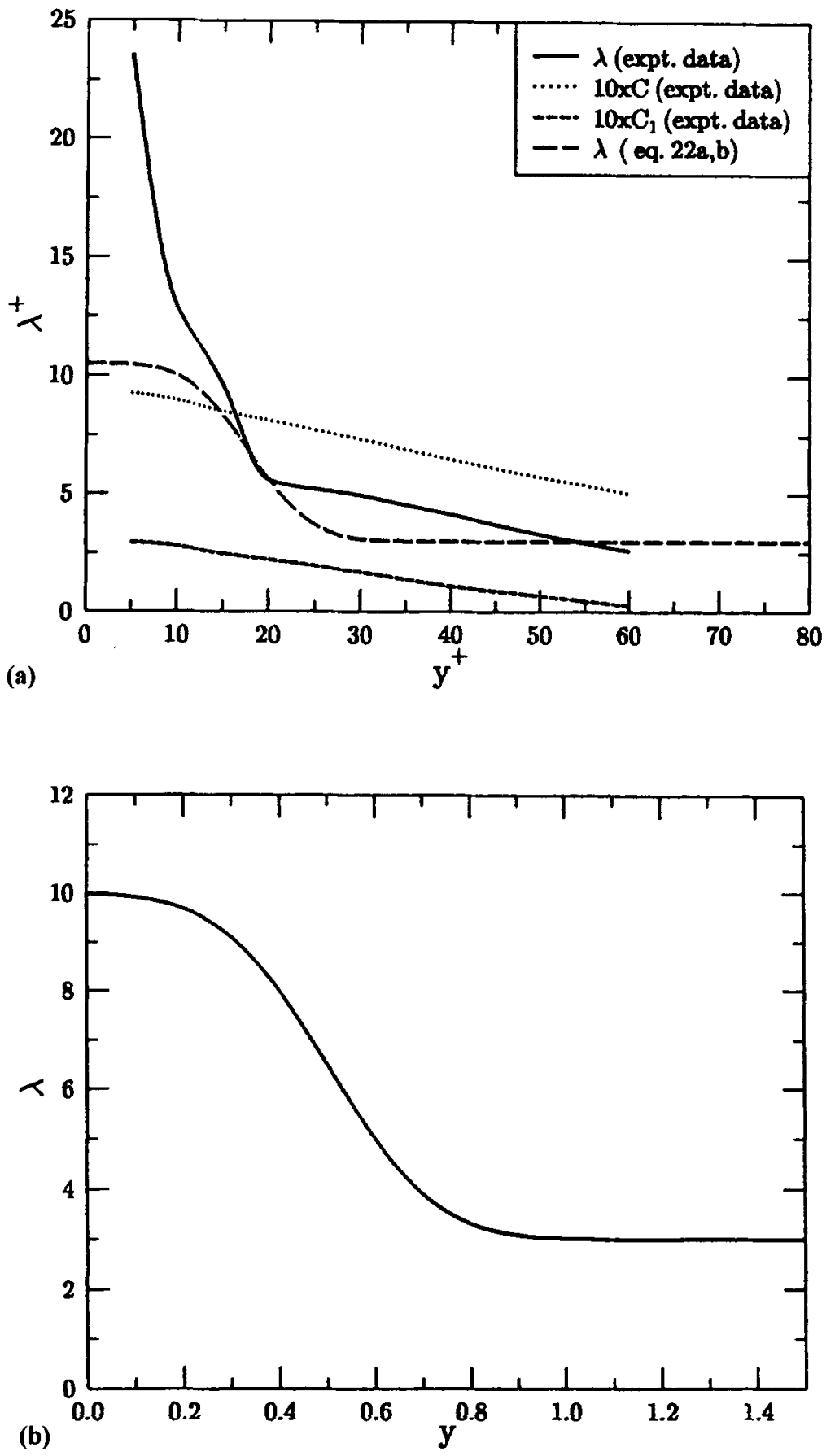

Figure 2. (a) Graph of anisotropy function $\lambda^{+}(=\lambda)$ versus $y^{+}$, standardised in terms of inner variables. Based on experimental data of Klebanoff, and from (22). Also shown are $C$ and $C_{1}$. (b) Graph of anisotropy function $\lambda$ versus $y\left(=y_{\mathrm{d}} / \delta\right)$, standardised in terms of outer variables, based on (23). 
the exact shape of the $\lambda$-curve is not important. Rather, instability is obtained with a high value of $\lambda(\simeq 9.0$ or more) in the wall region. Thus instability is due to the anisotropy in the wall region, a feature that could not have been captured by the isotropic eddy viscosity model.

Two of the forms used for $\lambda$ in the calculations are given below. The first is based on inner variables scaling and is given as follows:

$$
\begin{aligned}
& \lambda\left(y^{+}\right)=\lambda_{h i}\left[\lambda_{l o} / \lambda_{h i}+\left(\frac{1-\lambda_{l o} / \lambda_{h i}}{2}\right)\left\{1-\operatorname{erf}\left(\frac{y^{+}-y_{0 \lambda}^{+}}{\sigma_{\lambda}}\right)\right\}\right] \\
& \text { with, } \quad \lambda_{h i}=10.5 ; \quad \lambda_{l o}=3.0 ; \quad y_{0 \lambda}^{+}=18.0 ; \quad \sigma_{\lambda}=7.5 .
\end{aligned}
$$

The $\lambda$-curve based on (22) is shown in figure 2a. Equation (22) renders the stability equations stiff and the calculations are more laborious and constrained. This is because the region of high $\lambda$ is squashed close to the wall. For facility in calculation another expression for $\lambda$ was used based on outer variable scaling (plot shown in figure $2 b$ ) which gives a value of 10 at the wall relaxing much more gently to a value of 3 in the outer region. This equation is given as follows:

$$
\begin{aligned}
& \lambda(y)=\lambda_{h i}\left[\lambda_{l o} / \lambda_{h i}+\left(\frac{1-\lambda_{l o} / \lambda_{h i}}{2}\right)\left\{1-\operatorname{erf}\left(\frac{y-y_{0 \lambda}}{\sigma_{\lambda}}\right)\right\}\right] ; \\
& \text { with, } \quad \lambda_{h i}=10.0 ; \quad \lambda_{l o}=3.0 ; \quad y_{0 \lambda}=0.5 ; \quad \sigma_{\lambda}=0.25
\end{aligned}
$$

Other forms were also tried, varying the parameters in (23). The fact that instability is not dependent on one particular critical choice of the shape of the $\lambda$-curve, but is generally governed by high values of $\lambda$ in the wall region, is a point that greatly favours the statement of a general proposition: that instability is due to the anisotropy in the wall region.

\subsection{The disturbance equations}

We simplify the evolution equation (9) for $\tilde{u}_{i}$, remembering that the disturbances are twodimensional and Squire-transformable, and that the mean-flow is amenable to the quasiparallel approximation. A stream function $\psi$ is introduced for the organised disturbances such that $\tilde{u}=\partial \psi / \partial y$ and $\tilde{v}=-\partial \psi / \partial x$. After assuming normal modes, $\psi$ may be expressed in the following form:

$$
\psi=\phi(y) \exp [i \alpha(x-c t)],
$$

where, $\alpha$ is the spatial wavenumber and $c=c_{r}+i c_{i}$ is the (complex) wave speed. Introducing (14) in the evolution equation (9) for $\tilde{u}_{i}$, remembering the quasi-parallel approximation for the mean flow, and remembering the closure equations $(14,18)$ for $\tilde{r}_{i j}$, one arrives at extended forms of the Orr-Sommerfeld equation (a more detailed derivation may be found in Sen \& Veeravalli 1994). For the isotropic eddy viscosity model this is given as follows:

$$
\begin{aligned}
& i \alpha\left[(\bar{u}-c)\left(\phi^{\prime \prime}-\alpha^{2} \phi\right)-\bar{u}^{\prime \prime} \phi\right]-(1 / R)\left[\phi^{\prime \prime \prime \prime}-2 \alpha^{2} \phi^{\prime \prime}+\alpha^{4} \phi\right] \\
& \quad-(1 / R)\left[E\left\{\phi^{\prime \prime \prime \prime}-2 \alpha^{2} \phi^{\prime \prime}+\alpha^{4} \phi\right\}+2 E^{\prime}\left\{\phi^{\prime \prime \prime}-\alpha^{2} \phi^{\prime}\right\}\right. \\
& \left.\quad+E^{\prime \prime}\left\{\phi^{\prime \prime}+\alpha^{2} \phi\right\}\right]=0 .
\end{aligned}
$$


Equation (25) is identical to the equation obtained by Reynolds \& Hussain (1972), based on the Newtonian eddy model. The anisotropic eddy viscosity model induces further extensions over (25), due to the contribution of the $\lambda$-related terms. The extended OrrSommerfeld equation corresponding to the anisotropic eddy viscosity model is given as follows:

$$
\begin{aligned}
i \alpha & {\left[(\bar{u}-c)\left(\phi^{\prime \prime}-\alpha^{2} \phi\right)-\bar{u}^{\prime \prime} \phi\right]-(1 / R)\left[\phi^{\prime \prime \prime \prime}-2 \alpha^{2} \phi^{\prime \prime}+\alpha^{4} \phi\right] } \\
& -1 / R\left[E\left\{\phi^{\prime \prime \prime \prime}-2 \alpha^{2} \phi^{\prime \prime}+\alpha^{4} \phi\right\}+2 E^{\prime}\left\{\phi^{\prime \prime \prime}-\alpha^{2} \phi^{\prime}\right\}\right. \\
& \left.+E^{\prime \prime}\left\{\phi^{\prime \prime}+\alpha^{2} \phi\right\}\right]+(\lambda E / R)\left[-2 i \alpha \phi^{\prime \prime \prime}+2 i \alpha^{3} \phi^{\prime}\right] \\
& +\left(2 i \alpha \phi^{\prime} / R\right)\left[\lambda E^{\prime \prime}+2 \lambda^{\prime} E^{\prime}+\lambda^{\prime \prime} E\right]=0 .
\end{aligned}
$$

In both (25) and (26), primes $\left(^{\prime}\right)$ denote differentiation with respect to $y$. All quantities in (25) and (26) have been non-dimensionalised by $U_{\infty}, \delta$ and $\nu$, i.e. $E \equiv \epsilon / \nu, \bar{u} \equiv$ $\bar{u}_{\mathrm{d}} / U_{\infty}, y \equiv y_{\mathrm{d}} / \delta$ etc., where subscript 'd' represents dimensional or physical quantities. Also, in (25) and (26), the first group of terms in square brackets corresponds to the Rayleigh equation; the first two groups of terms in square brackets correspond to the classical Orr-Sommerfeld equation; and the remaining terms constitute the modifications in the classical Orr-Sommerfeld equation. Now, $E^{\prime}, E^{\prime \prime} \rightarrow 0$ (to be shown later) at the edge of the boundary layer, and, $\lambda$ can be made to obey the less stringent conditions $\lambda^{\prime}, \lambda^{\prime \prime} \rightarrow 0$ (without letting $\lambda \rightarrow 0$ ) at the edge of the boundary layer. Thus, the appropriate boundary conditions for the two extended Orr-Sommerfeld equations, viz. (25) and (26), are the same as those for the classical Orr-Sommerfeld equation, namely:

$$
\begin{aligned}
& \phi, \phi^{\prime}=0 \text { at } y=0 ; \\
& \phi(y) \sim e^{-\alpha y}, \text { for } y \rightarrow \infty .
\end{aligned}
$$

Either of the disturbance equations (25) or (26), along with the appropriate boundary conditions (27), constitutes an eigenvalue problem. In the temporal problem, $\boldsymbol{\alpha}$ and $\boldsymbol{R}$ are chosen real and $c=c_{r}+i c_{i}$ is determined as the (complex) eigenvalue. Stability or instability is obtained respectively as $c_{i}<0$ or $c_{i}>0$. As noted earlier the extended Orr-Sommerfeld equation, as given by (25), did not yield any unstable modes although near neutral modes were observed.

Next we look at the inner-variable form of (26). It is well known that at high values of $R$, the turbulence in the region close to the wall assumes a universal character, which is best seen by expressing the governing equation in inner variables. The velocity and length scales are $v_{*}$ and $v / v_{*}$, respectively. Denoting quantities scaled by inner variables by the superscript ( + , we note that, $\lambda=\lambda^{+} ; E=E^{+} ; B \equiv v_{*}^{2} / U_{\infty}^{2} ; y^{+}=y R \sqrt{B} ; \bar{u}^{+}=$ $\bar{u} / v_{*} ;$ and, $\alpha^{+}=\alpha /(R \sqrt{B})$, where $B$ is the non-dimensional wall shear stress. Thus, in inner variables, the disturbance equation (26) becomes:

$$
\begin{aligned}
& i \alpha^{+}\left[\left(\bar{u}^{+}-c^{+}\right)\left(\phi^{\prime \prime}-\alpha^{+^{2}} \phi\right)-\bar{u}^{+^{\prime \prime}} \phi\right]-\left[\phi^{\prime \prime \prime \prime}-2 \alpha^{+2} \phi^{\prime \prime}+\alpha^{+4} \phi\right] \\
& -\left[E^{+}\left\{\phi^{\prime \prime \prime \prime}-2 \alpha^{+^{2}} \phi^{\prime \prime}+\alpha^{+^{4}} \phi\right\}+2 E^{+^{\prime}}\left\{\phi^{\prime \prime \prime}-\alpha^{+^{2}} \phi^{\prime}\right\}\right. \\
& \left.+E^{+^{\prime \prime}}\left\{\phi^{\prime \prime}+\alpha^{+^{2}} \phi\right\}\right]+\lambda^{+} E^{+}\left[-2 i \alpha^{+} \phi^{\prime \prime \prime}+2 i \alpha^{+3} \phi^{\prime}\right] \\
& \quad+2 i \alpha^{+} \phi^{\prime}\left[\lambda^{+} E^{+^{\prime \prime}}+2 \lambda^{+^{\prime}} E^{+^{\prime}}+\lambda^{+^{\prime \prime}} E^{+}\right]=0 ;
\end{aligned}
$$

where, in (28), primes $\left(^{\prime}\right)$ denote differentiation with respect to $y^{+}$. Note that (28) does not show any explicit dependence on the Reynolds number, $R$. As will be seen later, (28) 
has a weak dependence on $R$ through the behaviour of $E^{+}$and $\lambda^{+}$, in the outer region. Nevertheless, if the unstable modes supported by equation (26) are wall modes, then the corresponding equation (28) would indicate that the behaviour of these wall modes should be near universal at high $R$. This is because the outer region does not have a strong influence on wall modes. Universality is actually confirmed by the present numerical results.

\section{Details of various functions}

The mean flow in wall turbulence is known to be multi-layered. Closest to the wall is the viscous sub-layer, followed by the buffer zone, and then followed respectively by the inner and outer log-law regions. Mathematical expressions are available for the mean velocity distributions, individually for each of these respective zones; but taken together these expressions do not constitute an analytically continuous expression for the mean velocity distribution. For hydrodynamic stability calculations as in the present problem, it is necessary to obtain an analytically continuous expression for the mean flow.

One very successful method of generating an analytically continuous mean velocity distribution for turbulent channel flow is reported in the work of Reynolds \& Tiederman (1967). In the present work, the mean velocity profile for turbulent boundary layer flow is generated on lines of the work of Reynolds \& Tiederman (1967). This requires obtaining suitable expressions for the eddy viscosity, and the shear stress distribution. This is discussed next.

\subsection{The expression for eddy-viscosity}

First we look at the eddy viscosity $E(y)$. The expression for the eddy viscosity $E(y)$ is given by a combined expression of Van Driest's law for the wall region and Reichardt's expression for the outer region. The composite $E(y)$ is given in Reynolds \& Tiederman (1967), and Reynolds \& Hussain (1972). Also, a slightly different expression is given in Hussain \& Reynolds (1975). The Reynolds \& Tiederman (1967) expression is given as follows:

$$
\begin{aligned}
E(y)= & \frac{1}{2}\left[1+\kappa^{2} \frac{R^{2} B}{9}\left(2 y-y^{2}\right)^{2}\left(3-4 y+2 y^{2}\right)^{2}\right. \\
& \left.\times\left\{1-\exp \left(\frac{-y R \sqrt{B}}{A^{+}}\right)\right\}^{2}\right]^{\frac{1}{2}}-\frac{1}{2} .
\end{aligned}
$$

In (29), $\kappa$ is the Von Karman constant, $A^{+}$is the Van Driest constant. The above equation is best suited for channel flow and a detailed plot of $E(y)$ may be seen in Reynolds \& Tiederman (1967). A slightly modified basic expression for $E(y)$ is needed for boundary layer flow, because $E^{\prime}, E^{\prime \prime} \simeq 0$ for $y=1$ (boundary layer edge). This basic expression, denoted by $E_{b}$, again needs to be further modified to account for the intermittency in the 
outer region. But first we define the basic expre ion $E_{b}$, as follows:

$$
\begin{aligned}
E_{b}(y)= & \frac{1}{2}\left[1+\kappa^{2} \frac{R^{2} B}{4}\left(2 y-y^{2}\right)^{2}\left(2-2 y+y^{2}\right)^{2}\right. \\
& \left.\times\left\{1-\exp \left(\frac{-y R \sqrt{B}}{A^{+}}\right)\right\}^{2}\right]^{\frac{1}{2}}-\frac{1}{2} .
\end{aligned}
$$

In the expression for $E_{b}$, i.e. in (30), $\kappa$ has been chosen as $\kappa=0.4$, and $A^{+}$has been chosen as $A^{+}=27$. Next we see in (30), what further modifications in $E_{b}$ are needed in order to accommodate the intermittency in the outer region. Although $E_{b}$ satisfies $E_{b}^{\prime}, E_{b}^{\prime \prime} \simeq 0$, at $y=1$, it still does not approach the right numerical value of $E$ at $y=1$. So, the first correction is to multiply $E_{b}$ by an error function $g(y)$, so that the right value of the eddy viscosity is obtained at $y=1$, prior to correction for intermittency. Next, to account for external intermittency of the boundary layer, for $y>0.4$, the eddy viscosity expression is further multiplied by the intermittency function (another error function) $\gamma(y)$. The final expression for eddy viscosity is given as follows:

$$
E(y)=E_{b}(y) g(y) \gamma(y)
$$

where,

$$
\begin{aligned}
& g(y)=(1-2 a)+a\left[1-\operatorname{erf}\left(\frac{y-y_{0 g}}{\sigma_{g}}\right)\right] \\
& \text { with, } \quad a=0.2 ; \quad y_{0 g}=0.4 ; \quad \sigma_{g}=0.13
\end{aligned}
$$

and,

$$
\begin{aligned}
& \gamma(y)=0.5\left[1-\operatorname{erf}\left(\frac{y-y_{0 \gamma}}{\sigma_{\gamma}}\right)\right] ; \\
& \text { with, } \quad y_{0 \gamma}=0.797 ; \quad \sigma_{\gamma}=0.204 .
\end{aligned}
$$

Plots of $E(y)$, for different values of $R$, and corresponding to (31), are shown in figure 3a. Also, figure $3 \mathrm{~b}$ shows the plot for $\epsilon /\left(v_{*} \delta\right)$ for $R=75,000$, and, shown in this figure also are the points corresponding to the measurements by Klebanoff (1954), reported in Hinze (1975).

One may look at the form of $E_{b}$ in inner variables, to make an assessment of the (marginal) departure from universality, of the disturbance equation (26) and (28) because of the outer scaling. Thus using inner scaling in (30) we have

$$
E_{b}^{+}=\frac{1}{2}\left[1+\kappa^{2} \frac{y^{+^{2}}}{4}(2-y)^{2}\left(2-2 y+y^{2}\right)^{2}\left\{1-\exp \left(\frac{-y^{+}}{A^{+}}\right)\right\}^{2}\right]^{\frac{1}{2}}-\frac{1}{2} \text {. }
$$

Note in (32) that $y \equiv y^{+} /(R \sqrt{B})$. Therefore, the outer region does contribute to $E_{b}^{+}$, and also therefore, $E_{b}^{+}$is dependent on the Reynolds number $R$. Nevertheless, as stated before, since this influence is in the outer region, this does not seriously affect the unstable 

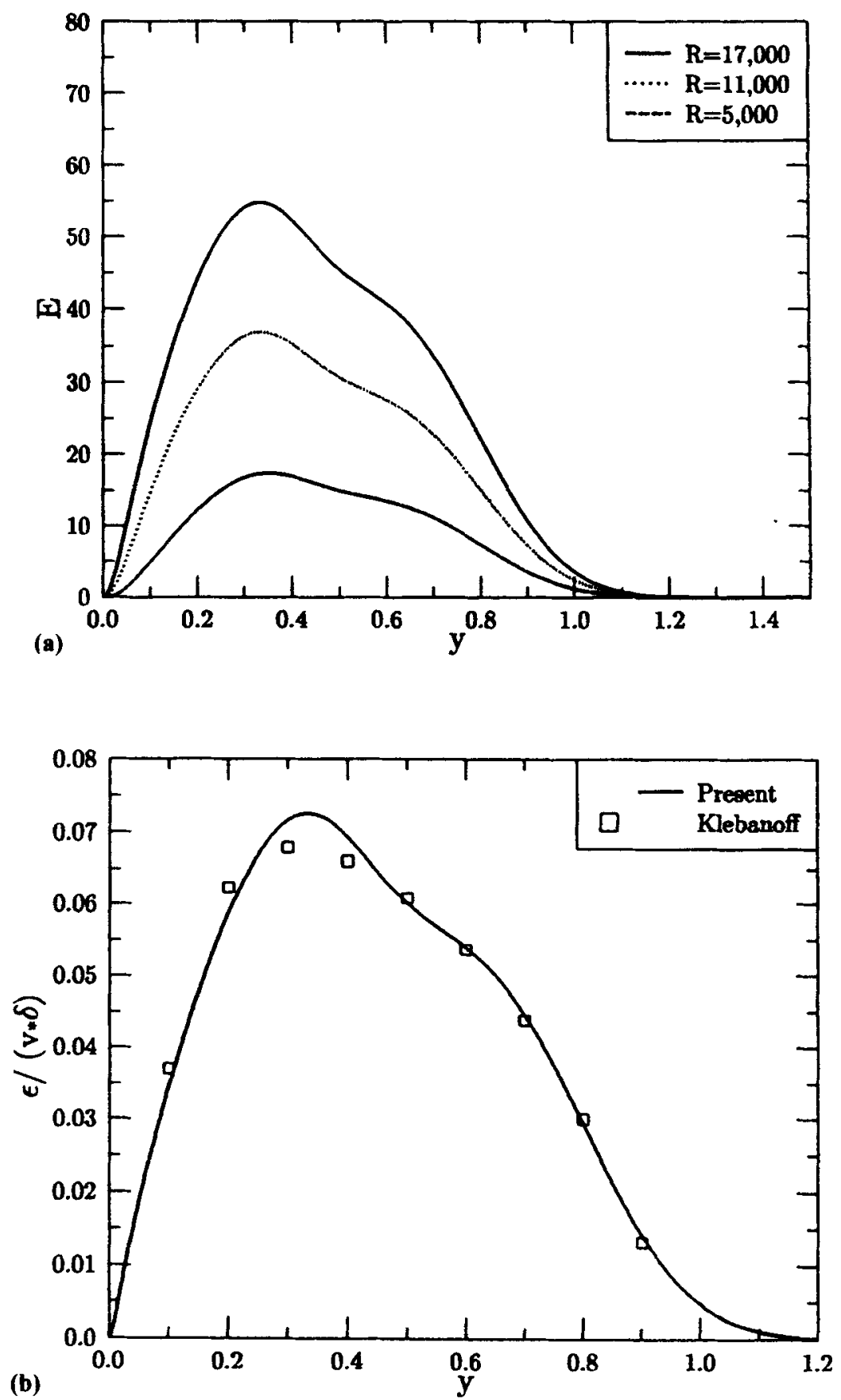

Figure 3. (Continued.) 


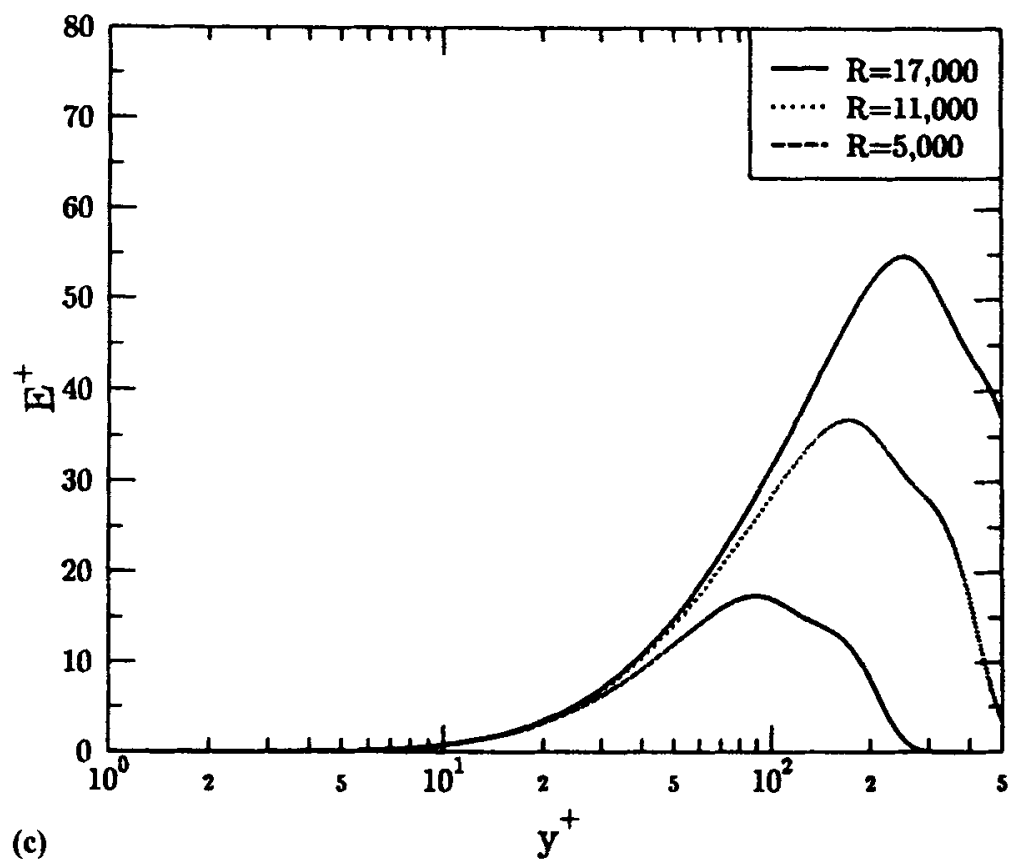

Figure 3. (a) Graph of eddy viscosity $E(=\epsilon / v)$ versus $y\left(=y_{\mathrm{d}} / \delta\right)$. (b) Graph of eddy viscosity $\epsilon /(v * \delta)$ versus $y\left(=y_{\mathrm{d}} / \delta\right)$. Comparison with Klebanoff's data at $\operatorname{Re}=75,000$. (c) Graph of eddy viscosity $E^{+}(=E)$ versus $y^{+}$, inner variables. Scale of $y^{+}$is logarithmic.

wall mode that is obtained in the problem. One may now similarly express (31) in inner variables scaling, to obtain $E^{+}\left(y^{+}\right)$(which is the same as $E\left(y^{+}\right)$). Figure $3 c$ shows plots of the eddy viscosity $E^{+}\left(y^{+}\right)$, in inner variables. This figure shows that in the inner region the eddy viscosity is the same across different $R$, a feature that is responsible for the universality of the wall modes.

\subsection{The expression for the shear stress distribution}

The distribution for the (total) shear stress $\tau$ is needed in order to be able to integrate the equation of motion to obtain the mean velocity distribution $\bar{u}(y)$. It is well known from experimental data that the distribution of mean shear looks similar to a tanh-profile, or a complementary error function profile. The expression chosen here is again a composite expression comprising an error function and a Gaussian function, to be able to match the experimental shear stress distribution, and, also, for the mean velocity profile to be able to satisfy the proper outer boundary conditions. This composite expression is given as follows:

$$
\tau(y)=\tau_{w} F(y) G(y) ;
$$

where, $\tau_{w}$ is the wall shear stress. Also $\tau$ is dimensional or non-dimensional depending on whether $\tau_{w}$ is dimensional or non-dimensional. When $\tau_{w}$ is non-dimensional then $\tau_{w}=B$. Further,

$$
\begin{aligned}
& F(y)=1+b\left[1+\operatorname{erf}\left(\frac{y-y_{0 F}}{\sigma_{F}}\right)\right] \\
& \text { with, } \quad b=0.7 ; \quad y_{0 F}=0.56 ; \quad \sigma_{F}=0.204 ;
\end{aligned}
$$




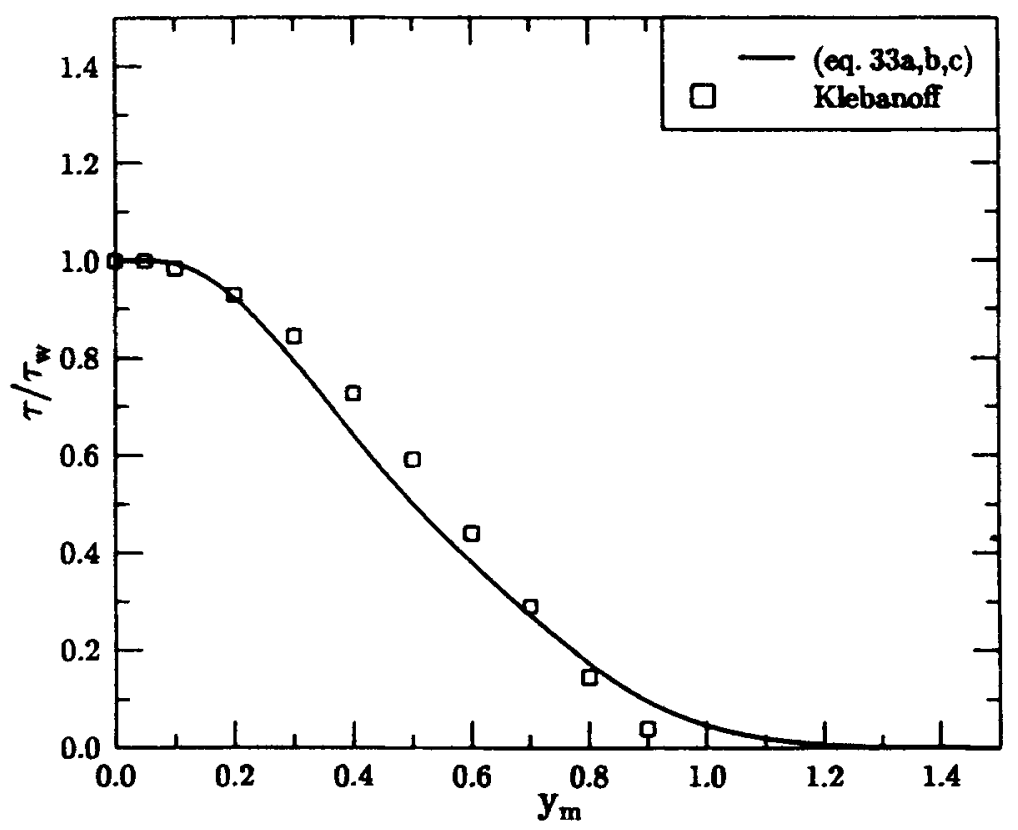

Figure 4. Graph of shear-stress $\tau / \tau_{w}$ versus $y_{m}$. Here $y$ is rescaled so that at $y_{m}=1$, $\bar{u}=0.99$. Data points of Klebanoff are also shown, for $\operatorname{Re}=75,000$.

and,

$$
\begin{aligned}
& G(y)=1, G^{\prime}(y)=0 ; \quad \text { for } 0 \leq y<y_{0 G} \\
& G(y)=\exp \left[-\frac{1}{2}\left(\frac{y-y_{0 G}}{\sigma_{G}}\right)^{2}\right] ; \quad \text { for } y \geq y_{0 G} \\
& \text { with, } \quad y_{0 G}=0.05 ; \quad \sigma_{G}=0.26
\end{aligned}
$$

A typical plot of $\tau / \tau_{w}$ versus $y$ is shown in figure 4 . In this figure the scale for $y$ is modified to $y_{m}$ (defined in the next sub-section). Also shown are data points for $\tau / \tau_{w}$ measured by Klebanoff (1954), and as reported in Hinze (1975).

\subsection{Generation of the mean velocity profile}

The $x$-momentum equation for mean velocity simplifies for boundary layer flow to the following:

$$
\bar{u} \frac{\partial \bar{u}}{\partial x}+\bar{v} \frac{\partial \bar{u}}{\partial y}=\frac{1}{R} \frac{\partial}{\partial y}\left[(1+E) \frac{\partial \bar{u}}{\partial y}\right]=\frac{\partial \tau}{\partial y},
$$

In the discussion that follows, the convective acceleration terms are not required. Hence (34) gives a relationship between $E$ and $\tau$ (which is in non-dimensional form). Thus, after integrating (at any given station in $x$ ), (34) becomes the following:

$$
(1+E) \frac{\partial \bar{u}}{\partial y}=R \tau
$$




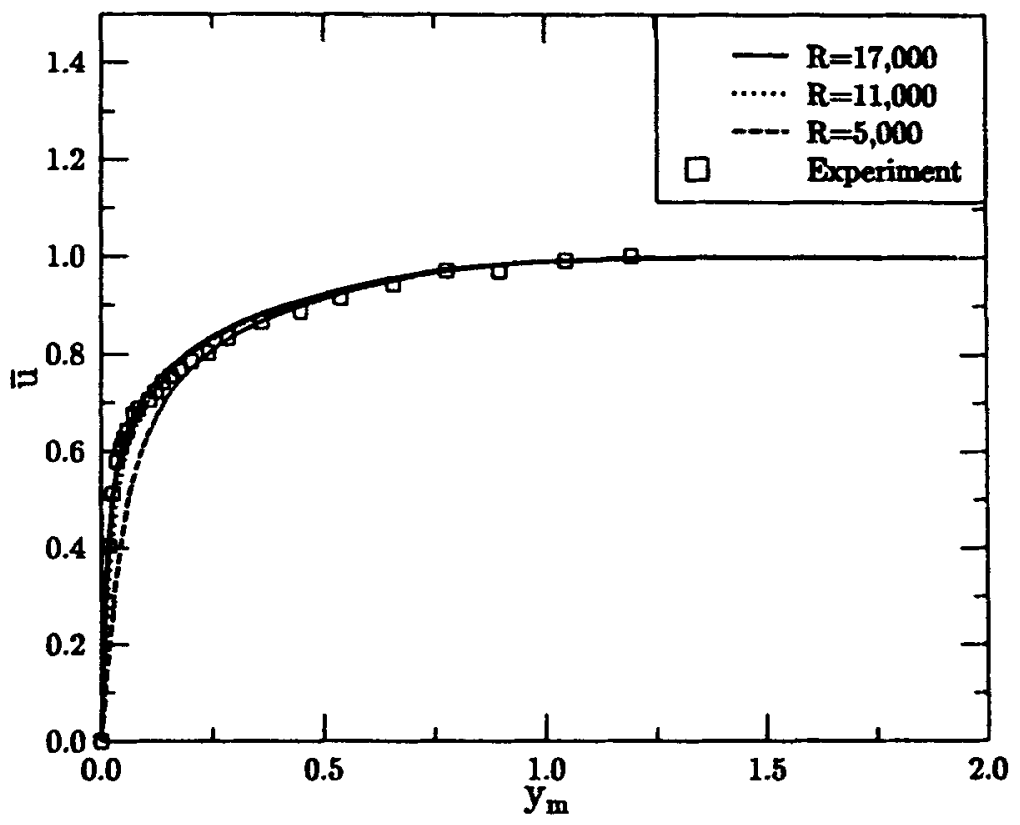

Figure 5. Graph of mean-velocity $u$ versus $y_{m} . y$ rescaled so that at $y_{m}=1, \bar{u}=0.99$. Data points are from present experiments, for $\operatorname{Re}$ around 17,000 .

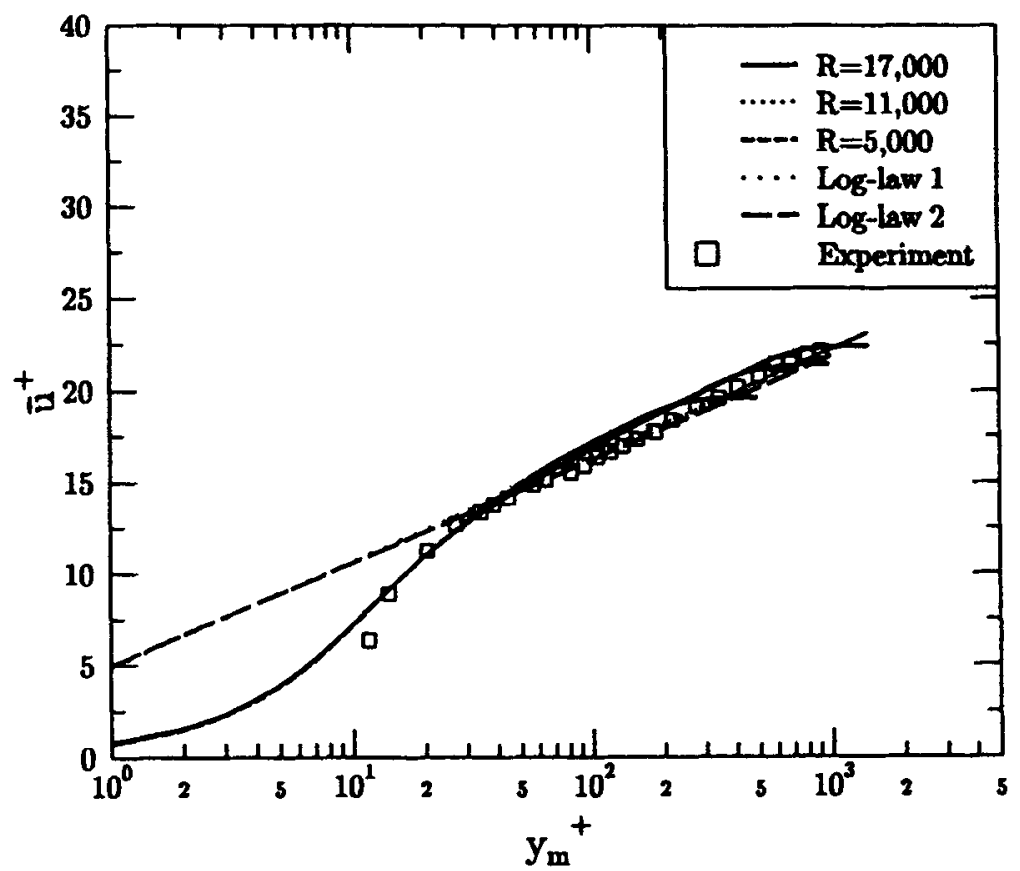

Figure 6. Graph of $\bar{u}^{+}$versus $y_{m}^{+}$. Also $y_{m}^{+}=y_{m} v * / \nu$, where $y$ is rescaled so that at $y_{m}=1$, $\bar{u}=0.99 . \log$-law $1: \bar{u}^{+}=5.0+5.75 \log \left(y^{+}\right)$. Log-law $2: \bar{u}^{+}=5.0+5.62 \log \left(y^{+}\right)$. 
where, in (35), $\tau$ is given from (33a,b,c). Also, it may be noted in (35) that $\partial \bar{u} / \partial y$ satisfies the condition $\partial \bar{u} / \partial y=R B$, for $y=0$. Also $\partial \bar{u} / \partial y$ satisfies the condition $\partial \bar{u} / \partial y \rightarrow 0$ for $y \rightarrow \infty$; because, $E \rightarrow 0$ for $y \rightarrow \infty$, and, the right hand side of (35), viz. $R \tau \rightarrow 0$ for $y \rightarrow \infty$. Integrating (35) we obtain

$$
\bar{u}=R B \int_{0}^{y} \frac{F\left(y^{\prime}\right) G\left(y^{\prime}\right)}{1+E\left(y^{\prime}\right)} \mathrm{d} y^{\prime} ;
$$

where, in (36), $F(y), G(y)$ are given from (33a,b,c). Since $\bar{u}$ must approach the free stream velocity $\bar{u}_{\infty}$ ( $=1$ in non-dimensional form), therefore, it follows from (36) that,

$$
1=R B \int_{0}^{\infty} \frac{F(y) G(y)}{1+E(y)} \mathrm{d} y .
$$

Thus iterating between (30), (31a,b,c), (33a,b,c), and (37) one obtains the converged value of $B$. After this the expression for $\bar{u}$ is given from equation (36).

Typical plots of the mean velocity distribution $\bar{u}$, both in outer and inner variables are shown respectively in figures 5 and 6 . Also shown in these figures are the points corresponding to the present experimental measurements. Solution for $\bar{u}$ from (36) is given in terms of $y\left(\equiv y_{\mathrm{d}} / \delta\right)$. Thus $y=1$ represents $y_{\mathrm{d}}=\delta$. Now, after $\bar{u}$ has been calculated from (36), it is not guaranteed that at $y=1, \bar{u}$ has the exact value of $\bar{u}=0.99$. Therefore, for comparison with experiments, it is sometimes expedient to rescale $y$ to $y_{m}$, where $y_{m} \equiv y / d_{99}$, and, for $y=d_{99}, \bar{u}=0.99$. The scale for $y$, in figures 5 and 6 , is thus modified to $y_{m}$, for ease of comparison with experimental results. Since the Reynolds numbers (in figures 5 and 6) are relatively small, the inner and outer regions are not well separated. Therefore, one need not expect the log-law to be valid over an extended region in $y_{m}^{+}$. This may also be confirmed from the DNS data of Spalart (see figure 5 of Spalart 1988). In figure 6 it may be noted that there is very good agreement in the inner-wall region. This is what is really important in the stability calculations because the critical point lies in the inner-wall region. In the outer region also, there is good agreement with the experimental data points.

It is clearly seen from the above figures that a very satisfactory method of generating an analytically continuous mean velocity has been obtained. Further refinements, if needed, may be made by adjusting the constants in the expressions for $E$ and $\tau$.

\section{Numerical results}

The numerical solutions of the extended Orr-Sommerfeld equation (26) were based on finite-difference methods developed in the earlier works of one of the authors (see Sen \& Vashist (1989) and Sen 1993). In the present problem, the methods described in the earlier works for the stability of the Blasius boundary layer are extended to the present turbulent boundary layer problem. The resolution used is ten times greater, and the basic step size in $y$, in the present problem, is $h=0.001$. Over a range of $0 \leq y \leq 1.5,1501$ points are required for each array specification. A seven-point finite difference scheme, employing a Noumerov transform and using a molecule described in Sen et al (1985), was employed in the calculations, and this gives basic round-off accuracy as $O\left(h^{6}\right)$, i.e. 


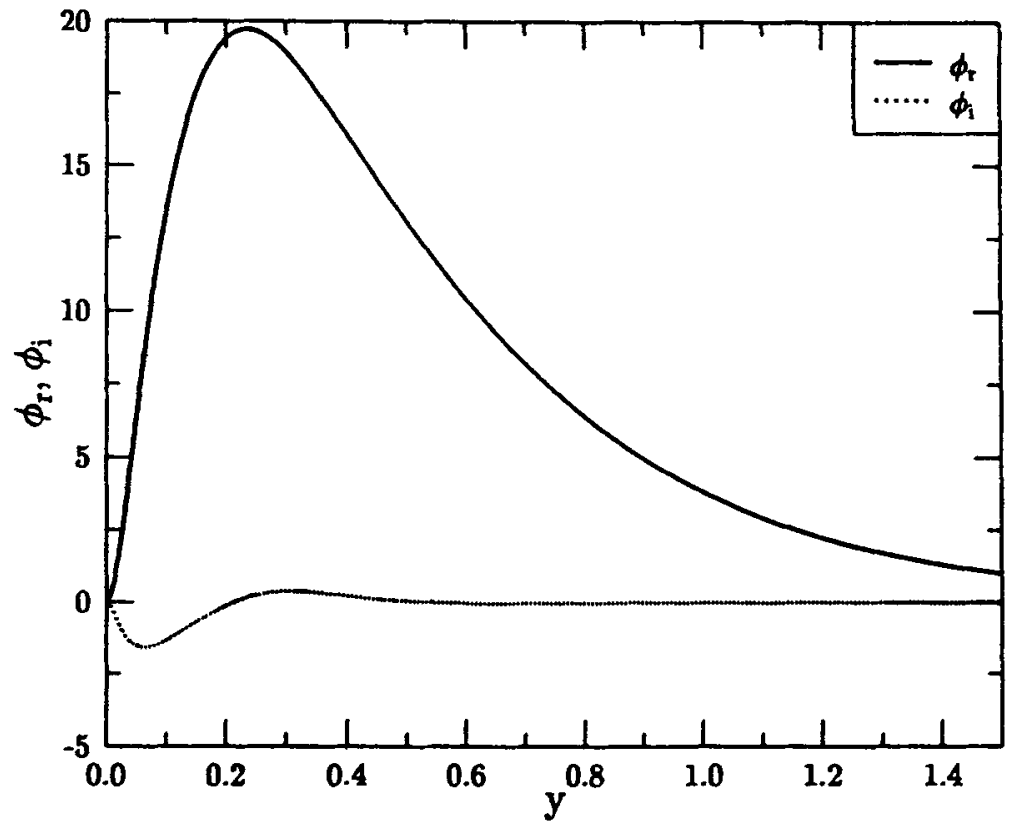

Figure 7. Graph of eigenfunction $\phi=\phi_{r}+i \phi_{i}$ versus $y$. Normalisation $\phi=1+0 i$ at $y=1.5$. Also $\operatorname{Re}=5000, \alpha=2.7, c_{r}=0.3486034, c_{i}=0.0044436$.

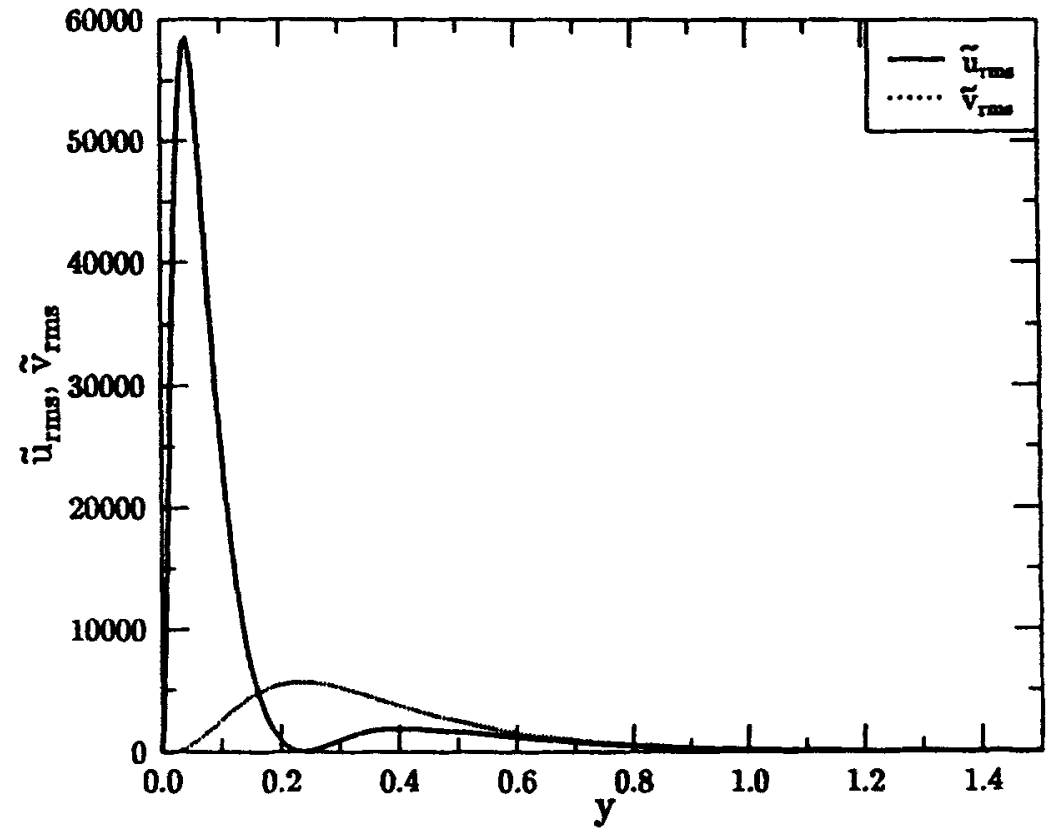

Figure 8. Graph of r.m.s. values of $\tilde{u}, \tilde{v}$ versus $y$. Normalisation, $\phi=1+0 i$ at $y=1.5$. Also, $\operatorname{Re}=5000, \alpha=2.7, c_{r}=0.3486034, c_{i}=0.0044436$. 


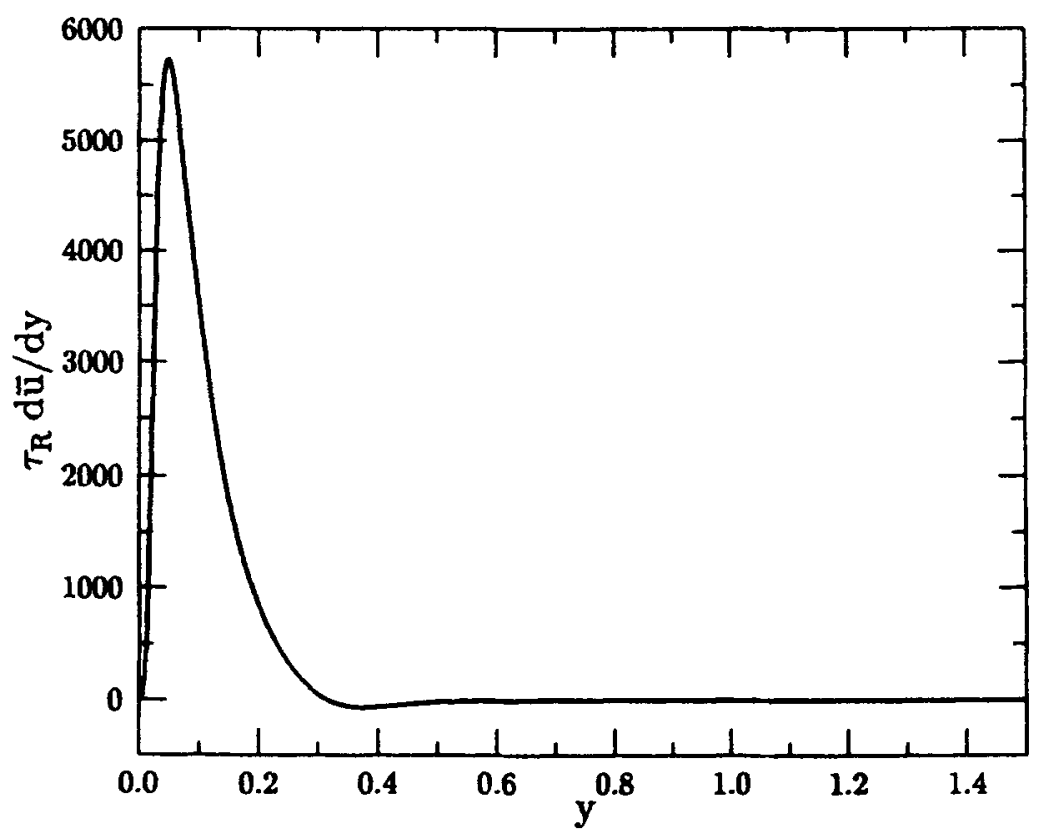

Figure 9. Graph of $\tau_{R} \mathrm{~d} \tilde{u} / \mathrm{d} y$ versus $y$. Normalisation, $\phi=1+0 i$ at $y=1.5$. Also, $\operatorname{Re}=5000, \alpha=2.7, c_{r}=0.3486034, c_{i}=0.0044436$.

$10^{-18}$ with $h=0.001$. Thus all care was taken to ensure that the very rapid changes taking place in the inner region, are faithfully taken into account in the numerical calculations. Also, the Blasius boundary layer results were verified as a cross-check. Calculations were performed using double precision arithmetic.

The numerical solution of the extended Orr-Sommerfeld equation (26) was obtained for a wide range of wavenumbers $\alpha$, for $R$ equal to 5000,11000 and 17000. At larger values of $R$ the sizes of the arrays become prohibitively large, if good resolution is desired close to the wall. However, from (28), it is clear that if one is interested in wall modes it is not really necessary to compute at very high $R$. Indeed, it is seen from the results presented below that trends become nearly universal in the reported range of $R$.

For all the three Reynolds numbers noted above, unstable eigenvalues were found for a wide range of $\alpha$. A typical set of results is shown in figures 7 and 8 , for the point defined by $(2.7,5000)$ in the $(\alpha, R)$ plane. Here, $c_{r}=0.349$ and $c_{i}=0.0044$. The value of $c_{r}$ indicates that this is a wall mode. (Note: $\bar{u}=0.35$ for $y=0.03$, when $R=5000$.) Figure 7 shows the eigenfunction $\phi$, normalised as $\phi=1+0 i$ at $y=1.5$. Figure 8 shows plots of the r.m.s. values of $\tilde{u}$ and $\tilde{v}$ as estimated from the numerical solution. As seen from figure 8 , r.m.s. values of $\tilde{u}$ peaks at $y^{+} \approx 11$, which agrees extremely well with experimental data (see Tennekes \& Lumley 1972) and the results of direct numerical simulations (Spalart 1988). Similar results were obtained for the production term, $\tau_{R}(\bar{u})^{\prime}$, where $\tau_{R}=\overline{\tilde{u}} \tilde{v}$, which peaks at $y^{+} \approx 12.5$, as shown in figure 9 . (The inordinately high values of ordinates in figures 8 and 9 are no cause for concern, and follow from the normalisation $\phi=1+0 i$ at $y=1.5$.)

Figure 10 shows the growth rate of the organised disturbance, $\alpha^{+} c_{i}^{+}$, plotted against the wavenumber $\alpha^{+}$. One sees that the band of unstable wavenumbers is fairly large and 


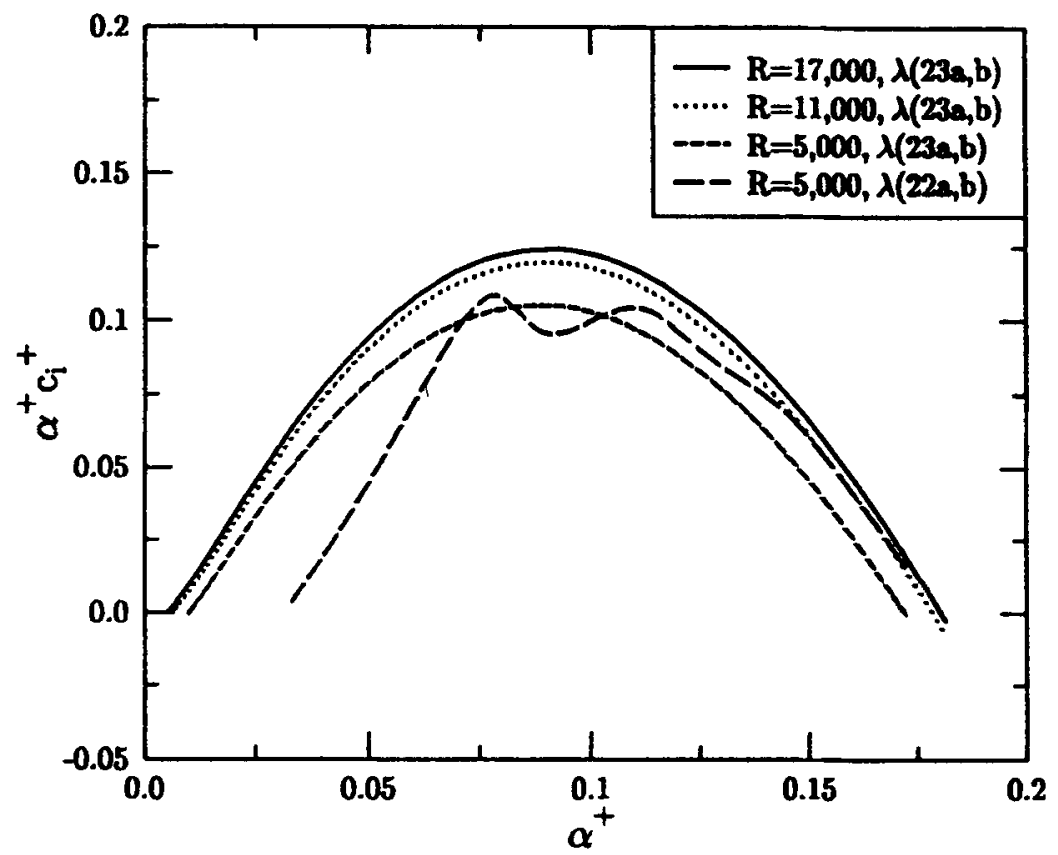

Figure 10. Graph of the growth rate, $\alpha^{+} c_{i}^{+}$versus $\alpha^{+}$, in inner variables scaling.

virtually the same, in inner variables, for all the three values of $R$. Secondly, in keeping with (26), the growth rate curves seem to reach, asymptotically, a limiting curve. Most of the calculations reported are with $\lambda$ based on (23). However, one growth rate curve is also shown in the figure, with $\lambda$ based on (22). As mentioned earlier, calculations using the latter expression of $\lambda$ are stiffer and therefore not persisted with for higher $R$. Nevertheless, the growth rate curves show that the trend of the results, and the range of unstable $\alpha$, are very comparable by these two widely different shapes of the $\lambda$-curves. Thus two important conclusions drawn are as follows. First, the instability is governed by a large value of $\lambda$ $(\simeq 9)$ in the inner region. Secondly, the exact shape of the $\lambda$-curve is not important.

\section{Experiments}

The experiments were conducted in the $0.61 \mathrm{~m} \times 0.61 \mathrm{~m} \times 4.75 \mathrm{~m}$ test section, suction tunnel of the Gas Dynamics Laboratory of this department. Figure 11 shows a schematic drawing of the tunnel and the coordinate system used. The boundary-layer growing along the bottom wall of the test section was chosen for measurement. A boundary-layer trip (1.5 mm square rod) was placed at the start of the test section. Other details on the tunnel and the instruments used for measurements may be found in Sen et al (1993)and Sen \& Veeravalli (1996a).

Measurements were made at a nominal tunnel speed of approximately $8 \mathrm{~m} / \mathrm{s}$ and at an $x$ location of $1.36 \mathrm{~m}$, along the centreline of the tunnel. Mean velocity measurements were made both with the pitot-tube and the hot-wire. The results are plotted in figures 5 and 6. However, only the hot-wire data is shown here as it enabled us to go much closer to 


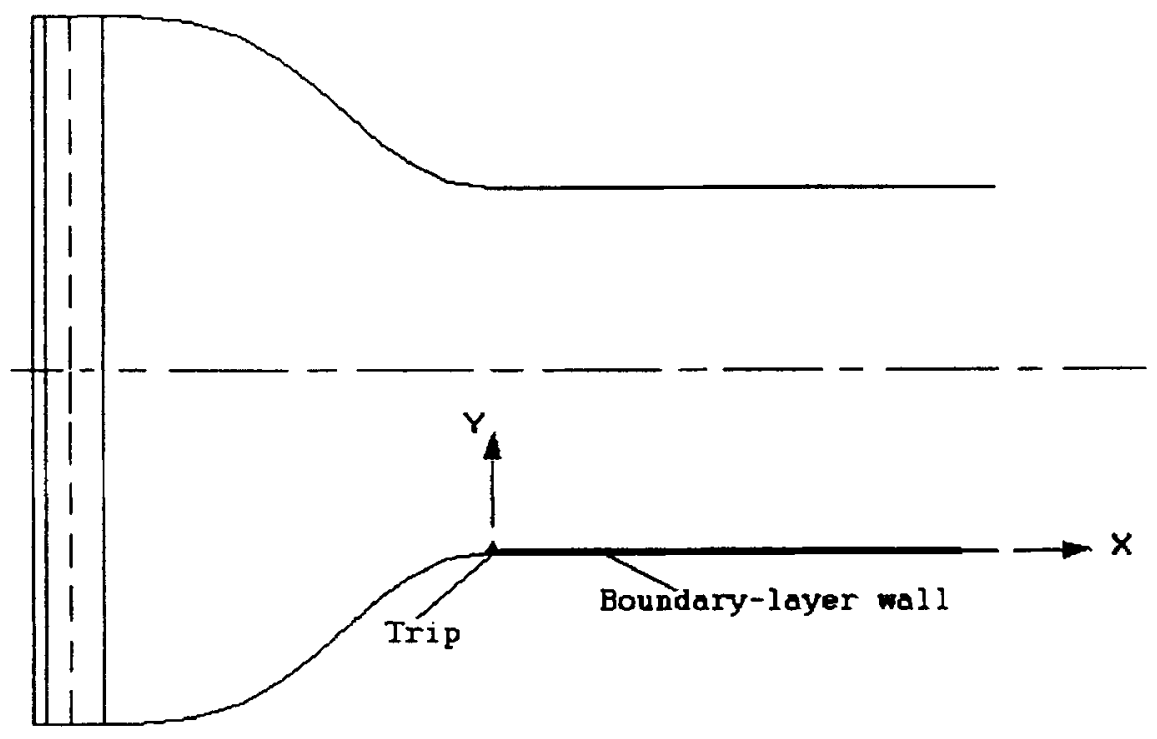

Figure 11. Schematic diagram of the experimental set-up.

the wall. The mean velocity profiles used in the numerical work and the best fit log-layer straight line from Purtell et al (1981) are also shown in figure 6. As mentioned before, since $R$ is not very high, the inner log-law region does not extend over a very wide range in $y^{+}$.

The friction velocity $v_{*}$ in the present experiments was estimated by several different methods. The best-fit log-layer straight line to various boundary layer experiments at comparable Reynolds numbers, reported in literature is (Purtell et al 1981):

$$
\bar{u}^{+}=5.0+5.62 \log _{10} y^{+} .
$$

where, $\bar{u}^{+} \equiv \bar{u} / v_{*}$ and $y^{+} \equiv y_{\mathrm{d}} v_{*} / v \equiv y R \sqrt{B}$.

The value of $v_{*}$ for the present experiments may thus be estimated from both the slope and the intercept of the above $\log$-law. From the slope one obtains $v_{*}=0.352 \mathrm{~m} / \mathrm{s}$ while from the intercept the value for $v_{*}$ is $0.353 \mathrm{~m} / \mathrm{s}$. The friction velocity may also be estimated from the Reynolds number $\boldsymbol{R}_{\theta}$, using the skin friction data reported in literature (Purtell et al 1981). Using this method one obtains a value of $0.344 \mathrm{~m} / \mathrm{s}$. Finally, the Preston-tube measurement using the calibration of Patel (1965) yielded $v_{*}=0.352 \mathrm{~m} / \mathrm{s}$. We see that

Table 1. Boundary-layer parameters.

$$
\begin{aligned}
& \delta^{*} \equiv \int_{0}^{\delta}\left(1-\bar{u} / U_{\infty}\right) \mathrm{d} y=4.61 \mathrm{~mm} \\
& \theta \equiv \int_{0}^{\delta} \frac{\bar{u}}{U_{\infty}}\left(1-\bar{u} / U_{\infty}\right) \mathrm{d} y=3.28 \mathrm{~mm} \\
& H \equiv \delta^{*} / \theta=1.41 \\
& R_{\theta} \equiv \frac{U_{\infty} \theta}{v}=1646
\end{aligned}
$$


the different methods for estimating the friction velocity agree to within $2.5 \%$. For the plots based on experiments in figures 5 and $6, v_{*}$ has been taken as $0.352 \mathrm{~m} / \mathrm{s}$.

The boundary layer thickness, defined for the experimental results as the location at which the mean velocity reaches $0.99 U_{\infty}$, is $3.35 \mathrm{~cm}$ for this $x$ location. Various parameters obtained from the mean velocity profile like the displacement thickness $\delta^{*}$ momentum thickness, $\theta$ etc. are reported in table 1 . The shape factor, $H$, falls well within the range reported in Purtell et al (1981) and agrees to better than 1\% with the data of Coles (1962), reported in Purtell et al (1981).

Figure 12 shows compensated, one-dimensional, power spectra of the $u^{\prime}$ velocity fluctuations, $\kappa^{+} E_{11}^{+}\left(\kappa^{+}\right)$, plotted against $\kappa^{+}$. Here $\kappa \equiv 2 \pi f / \bar{u}$, where $f$ is the frequency,

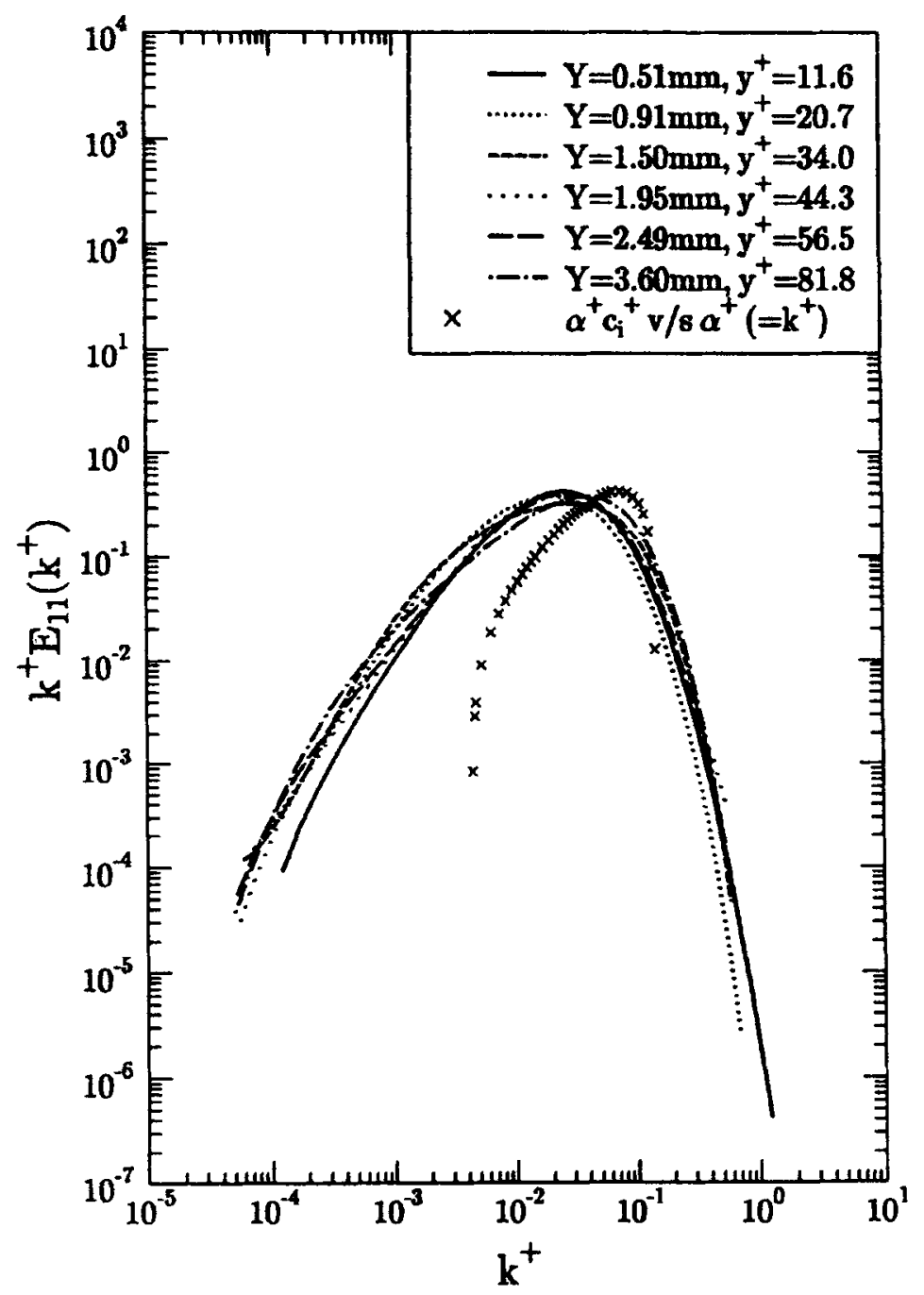

Figure 12. Graph of compensated power spectra $k^{+} E_{11}\left(k^{+}\right)$versus $k^{+}$, of the $u^{\prime}$ fluctuations, from present experiments. The points for $\alpha^{+} c_{i}^{+}$versus $k^{+}$are for $\operatorname{Re}=17,000$, and from stability calculations. Also, $\alpha^{+} c_{i}^{+}$is renormalised to have the same maximum value as the spectrum curve for $y^{+}=11.6$. 
and $\kappa^{+} \equiv \kappa /(R \sqrt{B})$. The spectra have been obtained at $y^{+}=11.6,20.7,34,44.3,56.5$ and 81.8 , i.e. in the buffer and $\log$ regions. All the spectra collapse reasonably well on to a single curve, as expected. Also shown in the same figure is the plot of the analytically obtained growth rate curve of $\alpha^{+} c_{i}^{+}$versus $\kappa^{+}$. Too much should not be read into the location of the 'peak' of this curve, as this curve only gives the growth rates according to linear stability theory. Nevertheless, the $\alpha^{+} c_{i}^{+}$versus $k^{+}$curve is contained fully within the collapsed experimental spectral curve, and within the energy-containing eddies region. This is yet another feature that keeps alive the possibility of some connection between the instability waves and actual turbulence.

\section{Conclusions}

An extended Orr-Sommerfeld equation has been derived to accurately describe the evolution of organised disturbances in the presence of background turbulence. This has been done based on a few novel features in the formulation. First it has been emphasized that the turbulent stresses in the problem can be suitably modelled based on the generalised eddy viscosity hypothesis (GEVH). This is a very significant observation to make, and this is the basis for legitimacy of the formulation of Reynolds \& Hussain (1972), as well as of the present formulation. Second, after stipulating the validity of GEVH in the present problem, a significant and logical improvement has been made in modelling the Reynolds stress tensor, over Reynolds \& Hussain (1972), based on the generalised anisotropic formulation of Pope (1975). This improvement is also unique in form. Thirdly, in the formulation, the anisotropy is properly accounted for in terms of a universal non-dimensional function $\lambda$, which has been called the 'anisotropy function' herein.

The numerical solution of the extended Orr-Sommerfeld equation (26) yielded unstable eigenvalues over a wide range of $\alpha$. It has been found that instability is obtained by a large value of $\lambda(\simeq 9$ or more) in the wall region. However, the calculations are not sensitive to the exact shape of the $\lambda$-curve. The mode of instability obtained is the wall-mode, and the eigenfunction and the r.m.s. distributions of $\tilde{u}$ and $\tilde{v}$ are found to be qualitatively similar to those obtained for Blasius flow. Moreover, the locations of the respective peaks of the $\tilde{u}$ r.m.s. profile and the production term $\tau_{R}(\bar{u})^{\prime}$, agree well with experimental values reported in literature, for actual turbulence. Thus the organised disturbances seem to mimic some of the features of actual turbulence.

The band of unstable eigenvalues, when scaled with respect to inner variables, indicates that results approach universality with high $R$. Particularly, the growth rate curves for different $R$, in inner variables, viz. $\alpha^{+} c_{i}^{+}$versus $\alpha^{+}$, approach a limiting curve for high $R$, as may be seen in figure 10 . This is also a feature that correlates well with the inner scaling in actual turbulence.

The experimental rig produces an adequately well-conditioned flat plate turbulent boundary layer flow to permit comparison with and validation of the numerical results. Detailed measurements of the one-dimensional power spectra for $u^{\prime}$ were taken at different $y^{+}$, in the buffer and $\log$ regions. These spectra were found to collapse well into a single curve. The limiting growth rate curve of the instabilities, plotted as $\alpha^{+} c_{i}^{+}$versus $\kappa^{+}$, is seen to be contained entirely within the power spectral curve of the $u^{\prime}$ fluctuations, and the 
entire range of unstable $\alpha^{+}$is contained within the energy containing eddies range. This is yet another feature that seems to support the possibility of some connection between the instabilities and actual turbulence.

As a final word in conclusion, it may be stated that the present work keeps alive the question of a possible connection between stability theory and actual turbulence, in wallbounded turbulent flows.

\section{Dedication}

We wish to dedicate this paper to Professor Peter Bradshaw FRS (somewhat belatedly) on the occasion of his 60th birthday. One of us (SVV) would also like to take this opportunity to thank Professor Bradshaw for all that he has learnt from him, through his books and lectures, and through discussions, on various theoretical and experimental aspects of turbulence, and on turbulent boundary layers in particular.

This project was funded by the Council of Scientific and Industrial Research, New Delhi on grant nos. 22(229) SP/92 EMR-II and 22(254)/96 EMR-II.

We would like to thank Mr G A Rakesh for his help in fabricating the boundary layer plate and for making the pitot-tube measurements. Lt A Bose, Mr Sharad K Gupta and Mr K Koren also helped in the measurements. The cooperation of Mr T R Bhogal and the other staff of the Gas Dynamics Laboratory is also gratefully acknowledged. Finally, we offer grateful thanks to Professor R Narasimha, FRS, for his constant encouragement, support and guidance.

\section{References}

Coles D F 1962 Rand Report R-403-PR, Rand Corp., Santa Monica, CA

Gaster M, Kit E, Wygnanski I 1985 Large-scale structures in a forced turbulent mixing layer. $J$. Fluid Mech. 150: 23-39

Hinze J O 1975 Turbulence 2nd edn (New York: McGraw-Hill)

Hussain A K M F, Reynolds W C 1970 The mechanics of an organised wave in turbulent shear flow. J. Fluid Mech. 41:241-258

Hussain A K M F, Reynolds W C 1972 The mechanics of an organised wave in turbulent shear flow. Part 2. Experimental results. J. Fluid Mech. 54: 241-261

Hussain A K M F, Reynolds W C 1975 Measurements in fully developed turbulent channel flow. J. Fluids Engg 97: 568-578

Jang P S, Benney D J, Gran R L 1986 On the origin of streamwise vortices in a turbulent boundary layer. J. Fluid Mech. 169: 109-123

Klebanoff P S 1954 Characteristics of turbulence in a boundary-layer with zero pressure gradient. NACA technical report No. 3178

Landau L D 1944 On the problem of turbulence. C. R. Acad. Sci. (URSS) 44: 311-314

Liu J TC 1988 Contributions to the understanding of large-scale coherent structures in developing free turbulent shear flows. Adv. Appl. Mech. 26: 183-309

Malkus W V R 1956 Outline of a theory of turbulent shear flow. J. Fluid Mech. 1: 521-539 
Patel V C 1965 Calibration of the Preston tube and limitations on its use in pressure gradients. $J$. Fluid Mech. 23: 185-208

Pope S B 1975 A more general effective viscosity hypothesis. J. Fluid Mech. 72: part 2: 331-340

Purtell L P, Klebanoff P S, Buckley F T 1981 Turbulent boundary-layer at low Reynolds number. Phys. Fluids 24: 802-811

Reynolds W C, Hussain A K M F 1972 The mechanics of an organized disturbance in turbulent shear flow. Part 3. Theoretical models and comparisons with experiments. J. Fluid Mech. 54: 263-288

Reynolds W C, Tiederman W G 1967 Stability of turbulent channel flow, with application to Malkus's theory. J. Fluid Mech. 27: 253-272

Roshko A 1992 Instability and turbulence in shear flows. Theoretical and applied mechanics (eds) S R Bodner, J Singer, A Solan, Z Hussain (Amsterdam: Elsevier)

Sen P K 1993 Some recent developments in the theory of boundary layer instability, Sādhana 18 : $387-403$

Sen P K, and Vashist T.K 1989 On the nonlinear stability of boundary-layer flow over a flat plate. Proc. R. Soc. London A424: 81-92

Sen P K, Venkateswarlu D, Maji S 1985 On the stability of pipe-Poiseuille flow to finite-amplitude axisymmetric and non-axisymmetric disturbances. J. Fluid Mech. 158: 289-316

Sen P K, Rakesh G A, Veeravalli S V 1993 Analysis of wall turbulence using hydrodynamic stability theory. Proc. 20th National Conf. on Fluid Mechanics and Fluid Power, Palghat, B3-1 to B3-6

Sen P K, Veeravalli S V 1994 Hydrodynamic stability theory applied to wall turbulence. Proc. 21st National Conf. on Fluid Mechanics and Fluid Power, Osmania University, Hyderabad, pp TF-1 to TF-6

Sen P K, Veeravalli S V 1996a Evolution of an organised disturbance in a turbulent boundary layer. Proc. 23rd National Conf. on Fluid Mechanics and Fluid Power, MACT, Bhopal, pp $1-12$

Sen P K, Veeravalli S V 1996b On a method of generating an analytically continuous turbulent mean velocity profile in turbulent boundary layer flow. Proc. 23rd National Conf. on Fluid Mechanics and Fluid Power, MACT, Bhopal, 13-18

Spalart P R 1988 Direct Numerical simulation of a turbulent boundary layer up to $R_{\theta}=1410 \mathrm{~J}$. Fluid Mech. 187: 61-98

Tennekes H, Lumley J L 1972 A first course in turbulence (Cambridge, MA: MIT Press)

Townsend A A 1976 The structure of turbulent shear flow 2nd edn (Cambridge: University Press) 